\title{
Responses of birds to large-scale wildfires in southern California
}

\author{
Lori Hargrove ${ }^{1, *}$ ANd Philip UnitT ${ }^{1}$
}

\begin{abstract}
In 2002 and 2003, $-4000 \mathrm{~km}^{2}$ of southern California burned in the region's largest fires in over a century. Within 1-2 years post-fire, we established 81 bird-survey routes (totaling over $200 \mathrm{~km}$ ) within the perimeters of the Pines, Cedar, and Coyote fires, and in nearby unburned habitat to serve as controls. We surveyed these routes repeatedly during both spring/ summer and winter for 5 years, with the objective of comparing species by pattern of response and identifying the species most strongly affected. Sampled habitat was primarily chaparral with isolated stands of oak woodland and coniferous forest. To quantify species' responses, we used data from the San Diego County Bird Atlas (1997-2002) as a pre-fire baseline, and used mixed-effects regression models to assess overall response to fire and post-fire trends. Responses and trends were diverse: in spring/summer, 24 species responded positively, 14 were neutral, and 43 responded negatively. In winter, 8 were positive, 18 neutral, and 34 negative. Some positive species (e.g., Lazuli Bunting, Passerina amoena) were strong "fire-followers," exploiting recently burned habitat, while others (e.g., Black-chinned Sparrow, Spizella atrogularis) increased mostly at higher elevations where forest was converted to chaparral. Neutral or "fire-resilient" species were either indifferent to fire (e.g., Ash-throated Flycatcher, Myiarchus cinerascens) or increased sharply after an initially negative response (e.g., Spotted Towhee, Pipilo maculatus). Among the negative or "fire-sensitive" species, recovery was often slow (e.g., California Thrasher, Toxostoma redivivum), and some continued to decline over time (e.g., Pygmy Nuthatch, Sitta pygmaea). By comparison with the pre-fire distribution as recorded in the atlas, the Rock Wren (Salpinctes obsoletus) was the strongest post-fire colonizer, while the Hutton's Vireo (Vireo huttoni) was the species most reduced. Small isolated breeding populations of the Red-breasted Sapsucker (Sphyrapicus ruber), Red-breasted Nuthatch (Sitta canadensis), Hermit Thrush (Catharus guttatus), Fox Sparrow (Passerella iliaca), and Goldencrowned Kinglet (Regulus satrapa) were eliminated from the Cuyamaca Mountains for at least the first 5 years after the fire. Annual rainfall during the study varied widely, slowing recovery in dry years. Variation in numbers of many species, especially granivorous winter visitors, paralleled variation in annual rainfall. If the area of southern California that burns annually increases as predicted, fire-followers should benefit, while species that are rare, patchily distributed, and dependent on later stages of succession will be most at risk. If the fires are a symptom of a shift toward a drier climate, they may cause a long-term or permanent reduction in numbers and ranges of fire-sensitive species.
\end{abstract}

Keywords: bird atlas, chaparral, Cuyamaca, fire, San Diego County

Supplemental Online Material for this paper (Table S1) is available at: www.wfopublications.org/Avifaunal_Change/Hargrove-Unitt/Hargrove-Unitt_SOM.pdf

$\mathrm{F}$ ire is a natural component of the ecology of southern California, and although the ranges of size, frequency, and intensity of fires under natural conditions are debated, humans have altered the regime and are faced with controversial management decisions. Increasingly frequent human-caused ignitions have led to more frequent

fires (Keeley et al. 1999), and with the climate warming, these fires are predicted to become more extensive and more severe, compounding the effects on species and ecosystems (McKenzie et al. 2004, Lenihan et al. 2008). Although fire management should be balanced with wildlife conservation, current conservation strategies in

Full citation: Hargrove, L., and Unitt, P. 2018. Responses of birds to large-scale wildfires in southern California, in Trends and traditions: Avifaunal change in western North America (W. D. Shuford, R. E. Gill Jr., and C. M. Handel, eds.), pp. 374-399. Studies of Western Birds 3. Western Field Ornithologists, Camarillo, CA; doi 10.21199/SWB3.20.

${ }^{1}$ San Diego Natural History Museum, P. O. Box 121390, San Diego, California 92112-1390; *corresponding author:

lhargrove@sdnhm.org 
southern California are based on very little biology with regard to fire (e.g., Regan et al. 2006).

Even though fire is integral to the ecology of chaparral, and an understanding of species' responses to fire is essential for good management, research on the response of chaparral birds to fire has been modest. Previous studies of avian fire ecology have taken place largely in commercially logged coniferous forests or other habitats not characteristic of southern California (Saab and Powell 2005, Leidolf and Bissonette 2009). Direct negative effects on birds, such as mortality of resident species and temporary loss of habitat, are to be expected from large-scale fires, but some species are more resilient than others, and some benefit from fire. Studies of avian response to fire in California shrublands have revealed such patterns as strong negative effects on chaparral-requiring species in the Sierra Nevada foothills (Lawrence 1966), greater diversity possible in burned chaparral (Wirtz 1979, 1982), general declines but individualistic responses in coastal sage scrub (McClure 1981, Moriarty et al. 1985, Stanton 1986), strong differences by site (Mendelsohn et al. 2008), and, after small-scale fire, greatest recovery of most species within the first few years (England 1995). In most studies, however, the limited number of sites has prevented generalization (e.g., Moriarty 2013), and sample sizes have tended to be too low for rigorous analysis of most species (e.g., Mendelsohn et al. 2008). The effects of large-scale fires on chaparral species have not been quantified broadly.

In 2002 and 2003, $-4000 \mathrm{~km}^{2}$ of southern California burned in the region's largest fires in over a century. In San Diego County, nearly $25 \%$ of the area covered by natural vegetation was burned. Most of this was chaparral, but also burned was nearly all the mixed coniferous/oak forest of the Cuyamaca Mountains, isolated from similar habitat on nearby mountains. Because of the unprecedented size of these fires, they were likely to have long-lasting effects, especially in the Cuyamaca Mountains. Therefore, over the 5 to 6 years following the 2002-2003 wildfires, we attempted to quantify these effects on birds by sampling consistently at multiple sites within the perimeters of three different fires and at comparable unburned sites, and by comparison to pre-fire data. Our objectives were to explore the diversity of post-fire responses through comparison of species, and to identify species that may be at risk from the increasing frequency of fires in southern California.

\section{METHODS}

\section{PRE-FIRE DATA}

Data for the San Diego County Bird Atlas (Unitt 2004) were recorded from March 1997 through February 2002 in the context of a grid defined by the public land survey consisting of more or less square cells averaging $4.8 \mathrm{~km}$ on a side. The primary objective, as is standard for bird atlases, was ascertaining breeding of each species by cell, but observers also recorded time spent in the field, counted or estimated numbers of each species per field survey, and described their area of coverage or sketched it on a field map. Observers were in the field through the spring and summer but most frequently in May and June. In addition, the San Diego County atlas had a winter (DecemberFebruary) component, based on observations and counts in the same spatial framework. In both the breeding season and winter, the goal for coverage of at least 25 hours in the field in each season was met in $97 \%$ of the grid squares. None of the squares in which the coverage goals were not met corresponded to areas surveyed post-fire.

\section{THE FIRES}

In July and August 2002, the Pines Fire burned 250 $\mathrm{km}^{2}$ mainly along the east slope of the Peninsular Ranges from near Hot Springs Mountain south almost to Mount Laguna (Figure 1). The following year in July, the Coyote Fire burned $75 \mathrm{~km}^{2}$ north of Hot Springs Mountain, and from 25 October to 3 November, the Cedar Fire burned $1134 \mathrm{~km}^{2}$ in central San Diego County, contiguous with the Pines Fire. Other fires in 2003, principally the Paradise and Otay fires, burned an additional 452 $\mathrm{km}^{2}$. The Cedar Fire was California's largest fire since at least 1889 (Keeley and Zedler 2009), and it swept large areas quickly, burning them at the highest level of severity (Keeley et al. 2008). In the isolated Cuyamaca Mountains, the fire consumed the forest canopy and killed virtually all conifers except within a few small enclaves, which Goforth and Minnich (2008) reported as covering 70 ha, less than $5 \%$ of the forest's previous extent.

\section{POST-FIRE SURVEYS}

We established a total of 81 survey routes (walked transects totaling over $200 \mathrm{~km}$ ), 56 in areas of San Diego County burned in the Pines, Coyote, and Cedar fires, and 25 in nearby unburned habitat to serve as controls (Figure 1). Routes fell within a total of 33 atlas squares and ranged from 716 to $1985 \mathrm{~m}$ in elevation, covering primarily chaparral 


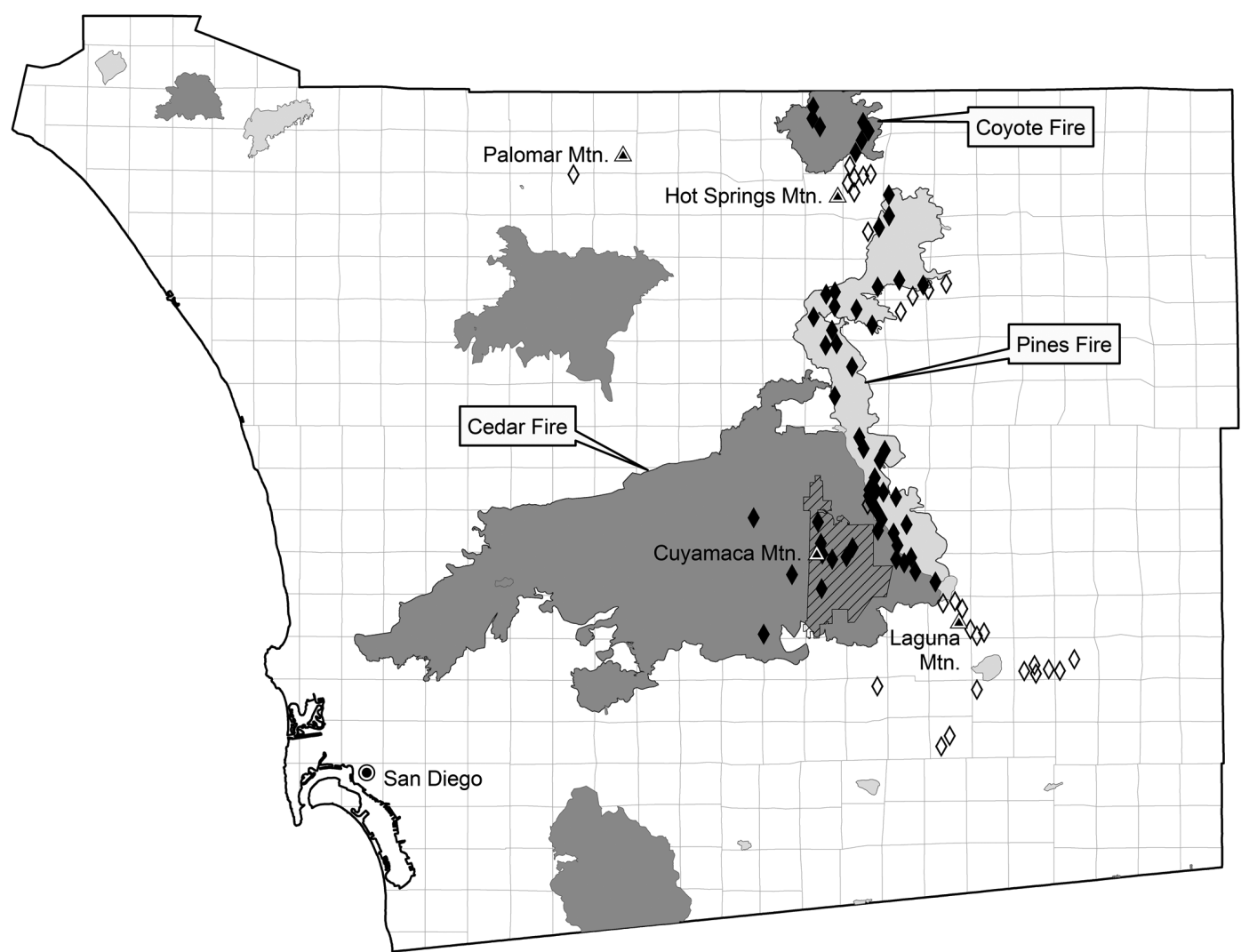

Figure 1. Perimeters of the San Diego County fires of 2002 (light gray) and 2003 (dark gray), and locations of survey routes in burned areas (black diamonds) and unburned areas (open diamonds). The bird atlas grid system is shown in the background (each square averages $4.8 \mathrm{~km}$ per side), and Cuyamaca Rancho State Park is indicated by fine cross-hatching.

and patches of oak/coniferous forest. We omitted coastal sage scrub at lower elevations, home to such rare or threatened species as the California Gnatcatcher (Polioptila californica) and San Diego Cactus Wren (Campylorhynchus brunneicapillus sandiegensis). Routes fell within areas managed by the Cleveland National Forest, Cuyamaca Rancho State Park, Anza-Borrego Desert State Park, Los Coyotes Indian Reservation, Cuyapaipe Indian Reservation, and Bureau of Land Management.

For every burned route or block of similar burned routes, we established control routes on the basis of proximity and similarity of habitat and elevation. Wherever possible, control routes were just outside a burned area (starting at the fire's perimeter), but some were up to $10 \mathrm{~km}$ away to match elevation and habitat. Because of the scarcity of birds on recently burned routes, we established more burned than control routes. To reduce any bias in post-fire trends, each route was typically surveyed by the same person over the 5 years. Almost all observers were experienced volunteers who had recently participated in the bird atlas, and these same observers typically covered nearby burned and unburned routes successively, maximizing comparability. Each route was between 1 and $4 \mathrm{~km}$ long and was walked in a single morning as an unlimited-distance transect, with all birds seen and heard along the route recorded. Each route was confined within a single atlas square and typically coincided with a route walked during the field work for the atlas (often by the same observer who covered the atlas square).

A primary set of 46 routes was surveyed on a very regular basis as described below, whereas surveys of a secondary set of 35 routes were less regular. The primary set was surveyed four times during the breeding season: once 15 April-7 May, once 8-31 May, once 1-21 June, and once 22 June-15 July. Also, each primary route was covered through the winter, once in December, once 
TABle 1. Post-fire survey effort after the Pines Fire (July-August 2002), Coyote Fire (July 2003), and Cedar Fire (October-November 2003).

\begin{tabular}{lccccc}
\hline & \multicolumn{2}{c}{ Number of routes } & & \multicolumn{2}{c}{ Years surveyed } \\
\cline { 2 - 3 } \cline { 5 - 6 } Survey set/Fire & Burned & Control & & Spring/summer & Winter \\
\hline Primary & & & & & \\
$\quad$ Pines & 31 & 5 & & $2003-2007$ & $2002-03-2006-07$ \\
Cedar (Cuyamaca) & 5 & 1 & & $2004-2008$ & $2004-05-2007-08$ \\
$\quad$ Cedar (Cleveland NF) & 3 & 1 & & $2005-2008$ & $2005-06-2007-08$ \\
Secondary & 5 & 6 & & $2005-2008$ & \\
$\quad$ Pines & 5 & 5 & & $2005-2008$ & \\
Cedar & 7 & 7 & & $2005-2008$ & \\
$\quad$ Coyote & & & & &
\end{tabular}

in January, and once in February. Each survey of a given route was separated by at least one week. These primary routes encompassed three subsets, one consisting of routes within the area burned in the Pines Fire, the other two within the area burned in the Cedar Fire, differing in managing agency and habitat (Table 1).

The primary Pines Fire subset of routes had much chaparral, often with patches of oak woodland and/or grassland; some were transitional between chaparral and desert scrub; a few skirted the east margins of stands of coniferous forest; two, along San Felipe and Banner creeks, had some riparian woodland. Elevation on these routes ranged from $716 \mathrm{~m}$ in San Felipe Valley to 1707 $\mathrm{m}$ on the east flank of Hot Springs Mountain. The Cedar Fire-Cuyamaca subset of routes surveyed areas that previously had been largely or entirely covered in mixed conifer/oak forest. Their elevations ranged from $1198 \mathrm{~m}$ at the Green Valley Falls campground to $1985 \mathrm{~m}$ on the summit of Cuyamaca Peak. Because the Cuyamaca Mountains burned so extensively, there was no area in those mountains where an unburned route of similar length could be established. Therefore, we established a single long control route $(5.5 \mathrm{~km})$ in Palomar Mountain State Park $50 \mathrm{~km}$ to the northwest. Its elevation ranged from 1359 to 1628 $\mathrm{m}$. The habitat along the Palomar route was also mixed conifer/oak forest but differed somewhat, especially in the prevalence on north-facing slopes of the big-cone Douglas fir (Pseudotsuga macrocar$p a)$, a tree absent from the Cuyamaca Mountains. Nevertheless, the pre-fire bird communities in the two areas were similar, and Palomar offered the best available parallel for comparison of post-fire trends. The Cedar Fire-Cleveland National Forest subset comprised three routes on the coastal slope (along or near Boulder Creek Road and Viejas Grade), plus one route in an unburned area (along Bear Valley Road south of Pine Valley). Elevations along the burned routes ranged from $823 \mathrm{~m}$ along the trail to Eagle Peak off Boulder Creek Road to $1199 \mathrm{~m}$ along Boulder Creek Road. Elevations along the unburned route ranged from $1183 \mathrm{~m}$ at the north end of Bear Valley Road to $1338 \mathrm{~m}$ at the top of the ridge east of Long Valley Peak. All four routes were similar in covering largely chaparral; the remaining $15-25 \%$ included groves of the coast live oak (Quercus agrifolia) or Engelmann oak (Q. engelmannii). From April 2005 through June 2008, Unitt surveyed all four of these routes.

A secondary set of 35 routes received fewer surveys on a more irregular schedule, but all were surveyed by Hargrove generally one to three times per year, from late spring to early summer, from 2005 to 2008 . They included 17 burned routes with elevations ranging from 1190 to $1692 \mathrm{~m}$, in the Coyote, Cedar, and Pines Fire areas, and 18 comparable control routes with elevations ranging from 960 to $1823 \mathrm{~m}$ (Table 1). Habitat covered an elevational gradient from desert-edge chaparral to montane chaparral to oak and conifer. These control routes included some higher-elevation forest (on Hot Springs and the Laguna mountains) that also served as good comparisons to Cuyamaca. The same protocol was followed as for the other routes, but, in addition, the distance from the transect line to the location of each bird was recorded, aided by laser rangefinder.

\section{Probability of Detection}

Probability of detection in burned vs. unburned habitat could be systematically biased if birds are more easily seen or heard in the more open burned habitat, but such bias is minimized in this study by long, repeated transects (high level of effort), skilled observers (atlas experience), and identification of species by voice. Nevertheless, we compared detectability in burned vs. unburned habitat on the basis of a subset of routes (the "secondary set," all by Hargrove) along which distances to 
TABLE 2. Detectability of common birds on burned and unburned routes ${ }^{a}$.

\begin{tabular}{|c|c|c|c|c|c|c|}
\hline \multirow[b]{2}{*}{ Species } & \multirow[b]{2}{*}{$n^{b}$} & \multirow[b]{2}{*}{$D_{\max }(\mathrm{m})^{c}$} & \multicolumn{2}{|c|}{ In burned } & \multicolumn{2}{|c|}{ Difference in unburned } \\
\hline & & & $D_{\text {avg }}(\mathrm{m})^{d}$ & $P^{e}$ & $D_{\text {avg }}(\mathrm{m})^{f}$ & Pg \\
\hline Anna’s Hummingbird & 88 & 60 & 22.2 & 0.09 & 0.0 & 0.00 \\
\hline White-breasted Nuthatch & 40 & 125 & 43.0 & 0.23 & 4.1 & 0.06 \\
\hline California Towhee & 148 & 145 & 52.1 & 0.22 & 9.0 & 0.05 \\
\hline Bewick's Wren & 198 & 160 & 54.2 & 0.23 & -3.0 & -0.02 \\
\hline Lazuli Bunting & 193 & 160 & 41.8 & 0.20 & -1.2 & 0.02 \\
\hline Spotted Towhee & 396 & 170 & 48.2 & 0.23 & 3.2 & 0.02 \\
\hline California Scrub-Jay & 208 & 200 & 72.0 & 0.29 & 17.9 & 0.08 \\
\hline Black-chinned Sparrow & 491 & 200 & 55.5 & 0.24 & 4.4 & 0.02 \\
\hline California Thrasher & 67 & 200 & 62.5 & 0.31 & -5.3 & -0.03 \\
\hline Mountain Quail & 201 & 300 & 96.3 & 0.48 & 1.7 & -0.02 \\
\hline Average & & & 54.8 & 0.25 & 3.1 & 0.02 \\
\hline
\end{tabular}

${ }^{a}$ As measured by distance sampling in the mountains of San Diego County, 2005-2008.

${ }^{b}$ Total number of observations, based on 152 surveys (74 in unburned and 78 in burned) along 35 routes.

${ }^{c}$ Maximum distance of detection recorded.

${ }^{d}$ Average distance of detection.

${ }^{e}$ Probability of detection of a bird within a perpendicular distance of $300 \mathrm{~m}$.

$f$ Difference in average distance of detection.

$g$ Difference in probability of detection within $300 \mathrm{~m}$.

each bird were recorded by laser rangefinder. For analysis, we pre-selected 10 species for which we had large samples. For the distance analysis we used program $\mathrm{R}$ (version 3.1.0) with the package Unmarked (Fiske and Chandler 2011) with the half-normal detection function in the routine "distsamp." On the basis of a separate model for each species, we estimated the probability of detection within $300 \mathrm{~m}$ (maximum distance recorded) in both burned and unburned habitat.

Across the 10 species tested, the distance of detection averaged only $3 \mathrm{~m}$ greater along burned routes than along unburned routes (Table 2). The average difference between burned and unburned routes in probability that a bird present within $300 \mathrm{~m}$ would be detected was $\Delta p=0.02$, ranging from -0.03 for the California Thrasher to +0.08 for the California Scrub-Jay (Table 2; see Table 3 for scientific names). Therefore, we used a difference in abundance of at least $10 \%$ as a minimum threshold to categorize a species as responding positively or negatively to fire.

\section{ANALYSIS OF RESPONSE TO FIRE AND POST-FIRE TRENDS}

We excluded from the analysis water birds, nocturnal birds, and species occurring in the region only during migration. For the spring/summer season, we excluded all records prior to 8 May for a species if spring migrants passing through at that time substantially augment the breeding population. Seventeen species had data excluded for this reason. We excluded data for a species if its primary habitat had not been present before the fire along the routes covered after the fire. The latter exclusion affected mainly riparian and grassland species, because these habitats tended to be patchy within an atlas square, thus not always well represented by the survey route. We also excluded a survey route from analysis for a species if it was both outside its main elevational range (not within the first and third quartiles of its post-fire elevational distribution), and the species never occurred on the route pre-fire or post-fire. After exclusions, we further required a minimum of 25 records (any observation of the species during a survey regardless of number, with at least 3 records in at least 3 different years, and at least 3 qualifying routes in both burned and unburned (control) habitat.

The total number of surveys was 1017 in spring/summer and 627 in winter. For abundance, we calculated the number of birds per kilometer for each species and survey. We modeled the relationship between abundance (birds/ $\mathrm{km}$ ) and fire for each species in each season by using $\mathrm{R}$ and lme4 (Bates et al. 2014) for linear mixedeffects analysis with function "lmer" and ordinary (not restricted) maximum-likelihood estimation (Burnham and Anderson 2002). Rather than use a model-selection approach, we constructed the same single model for each species in each season so we could compare responses to fire and postfire trends across species. Variables functioning as main fixed effects were burned or unburned ("Burned," value yes or no) and year since fire 
TABLE 3. Bird responses to large-scale fire in San Diego County, California, species listed from positive to negative ${ }^{a}$.

\begin{tabular}{|c|c|c|c|c|c|c|c|c|c|c|}
\hline \multirow[b]{2}{*}{ Species and season ${ }^{b}$} & \multirow[b]{2}{*}{ Routes $^{c}$} & \multirow[b]{2}{*}{$\mathrm{PPT}^{d}$} & \multicolumn{3}{|c|}{ Burned $^{e}$} & \multicolumn{3}{|c|}{ Burned $\times$ Year $f$} & \multirow[b]{2}{*}{$\mathrm{FRI}^{g}$} & \multirow[b]{2}{*}{$\begin{array}{c}\text { FRI } \\
\text { trend }\end{array}$} \\
\hline & & & $\begin{array}{l}\text { Coef- } \\
\text { ficient }\end{array}$ & SE & $p$ & $\begin{array}{l}\text { Coeffi- } \\
\text { cient }\end{array}$ & SE & $p$ & & \\
\hline $\begin{array}{l}\text { Dusky Flycatcher (Empidonax } \\
\text { (Eherholeri: DUFL). }\end{array}$ & 13 & CYP & 0.15 & 0.21 & 0.41 & 0.03 & 0.04 & 0.52 & 0.68 & 0.25 \\
\hline $\begin{array}{l}\text { White-throated Swift (Aeronautes } \\
\text { saxatalis; WTSW): spring/summer }\end{array}$ & 76 & PYP & 0.17 & 0.11 & 0.10 & 0.07 & 0.04 & 0.06 & 0.59 & 0.52 \\
\hline $\begin{array}{l}\text { Lazuli Bunting (Passerina amoena; } \\
\text { LAZB): spring summer }\end{array}$ & 81 & CYP & 1.96 & 0.63 & 0.00 & -0.61 & 0.18 & 0.00 & 0.58 & -0.34 \\
\hline \multicolumn{11}{|l|}{$\begin{array}{l}\text { Rock Wren (Salpinctes obsoletus; } \\
\text { ROWR) }\end{array}$} \\
\hline spring/summer & 81 & CYP & 0.77 & 0.26 & 0.00 & -0.23 & 0.07 & 0.00 & 0.57 & -0.32 \\
\hline winter & 42 & PYP & 0.22 & 0.27 & 0.40 & -0.04 & 0.07 & 0.53 & 0.36 & -0.13 \\
\hline $\begin{array}{l}\text { Costa's Hummingbird (Calypte costae; } \\
\text { COHU): spring/summer }\end{array}$ & 81 & CYP & 0.46 & 0.17 & 0.01 & 0.01 & 0.06 & 0.90 & 0.44 & 0.01 \\
\hline $\begin{array}{l}\text { Black-chinned Sparrow (Spizella } \\
\text { atrogularis; BCSP): spring/summer }\end{array}$ & 81 & PYP & 1.44 & 0.53 & 0.01 & 0.31 & 0.15 & 0.03 & 0.34 & 0.15 \\
\hline $\begin{array}{l}\text { Canyon Wren (Catherpes mexicanus; } \\
\text { CANW): spring/summer }\end{array}$ & 64 & CYP & 0.02 & 0.03 & 0.46 & 0.01 & 0.01 & 0.25 & 0.31 & 0.18 \\
\hline $\begin{array}{l}\text { Northern Harrier (Circus cyaneus; } \\
\text { NOHA): winter }\end{array}$ & 38 & PYP & 0.02 & 0.02 & 0.43 & 0.01 & 0.01 & 0.60 & 0.28 & 0.22 \\
\hline \multicolumn{11}{|l|}{ European Starling (Sturnus vulgaris; } \\
\hline spring/summer & 75 & CYP & 0.29 & 0.36 & 0.42 & -0.13 & 0.11 & 0.20 & 0.26 & -0.22 \\
\hline winter & 44 & PYP & 0.32 & 0.54 & 0.54 & 0.10 & 0.17 & 0.53 & 0.30 & 0.21 \\
\hline \multicolumn{11}{|l|}{ Chipping Sparrow (Spizella passerina; } \\
\hline spring/summer & 61 & CYP & 0.08 & 0.09 & 0.40 & -0.01 & 0.04 & 0.75 & 0.36 & -0.11 \\
\hline winter & 35 & PYP & 0.51 & 0.44 & 0.22 & -0.10 & 0.21 & 0.63 & 0.11 & -0.04 \\
\hline $\begin{array}{l}\text { Lawrence's Goldfinch (Spinus } \\
\text { lawrencei; LAGO): spring summer }\end{array}$ & 80 & CYP & 0.38 & 0.45 & 0.39 & -0.48 & 0.16 & 0.00 & 0.22 & -0.44 \\
\hline \multicolumn{11}{|l|}{$\begin{array}{l}\text { Red-tailed Hawk (Buteo jamaicensis; } \\
\text { RTHA) }\end{array}$} \\
\hline spring/summer & 79 & PYP & 0.18 & 0.07 & 0.02 & 0.04 & 0.02 & 0.05 & 0.37 & 0.20 \\
\hline winter & 45 & PYP & 0.06 & 0.10 & 0.54 & 0.05 & 0.03 & 0.15 & 0.07 & 0.13 \\
\hline $\begin{array}{l}\text { Western Kingbird (Tyrannus verticalis; } \\
\text { WEKI): spring/summer }\end{array}$ & 67 & CYP & 0.04 & 0.08 & 0.54 & -0.05 & 0.03 & 0.06 & 0.21 & -0.36 \\
\hline \multicolumn{11}{|l|}{ Mourning Dove (Zenaida macroura; } \\
\hline spring/summer & 81 & CYP & 0.83 & 0.69 & 0.22 & -0.32 & 0.24 & 0.17 & 0.17 & -0.12 \\
\hline winter & 45 & PYP & 0.56 & 1.35 & 0.66 & 0.67 & 0.47 & 0.15 & 0.20 & 0.69 \\
\hline \multicolumn{11}{|l|}{ American Kestrel (Falco sparverius; } \\
\hline $\begin{array}{l}\text { AMKE) } \\
\text { spring/summer }\end{array}$ & 77 & PYP & 0.05 & 0.04 & 0.18 & 0.02 & 0.02 & 0.25 & 0.16 & 0.13 \\
\hline winter & 46 & PYP & 0.03 & 0.03 & 0.33 & 0.02 & 0.02 & 0.21 & 0.17 & 0.26 \\
\hline $\begin{array}{l}\text { Lincoln’s Sparrow (Melospiza lincolnii; } \\
\text { LISP): winter }\end{array}$ & 41 & PYP & 0.12 & 0.14 & 0.37 & -0.01 & 0.05 & 0.85 & 0.16 & -0.02 \\
\hline \multicolumn{11}{|l|}{ Lesser Goldfinch (Spinus psaltria; } \\
\hline spring/summer & 81 & PYP & 0.97 & 0.50 & 0.05 & 0.13 & 0.14 & 0.38 & 0.27 & 0.07 \\
\hline winter & 46 & PYP & 0.08 & 0.32 & 0.80 & 0.07 & 0.16 & 0.65 & 0.04 & 0.10 \\
\hline $\begin{array}{l}\text { Bell's Sparrow (Artemisiospiza belli; } \\
\text { SAGS): spring/summer }\end{array}$ & 74 & PYP & 0.07 & 0.10 & 0.44 & 0.11 & 0.04 & 0.01 & 0.12 & 0.51 \\
\hline \multicolumn{11}{|l|}{ Say's Phoebe (Sayornis saya; SAPH) } \\
\hline spring/summer & 54 & CYP & 0.01 & 0.03 & 0.81 & 0.00 & 0.01 & 0.99 & 0.09 & -0.01 \\
\hline winter & 32 & PYP & 0.01 & 0.05 & 0.78 & 0.02 & 0.02 & 0.25 & 0.10 & 0.36 \\
\hline
\end{tabular}

House Finch (Haemorhous mexicanus; HOFI) 
TABLE 3 (continued).

\begin{tabular}{|c|c|c|c|c|c|c|c|c|c|c|}
\hline \multirow[b]{2}{*}{${ }_{\text {Species and season }}^{b}$} & \multirow[b]{2}{*}{ Routes $^{c}$} & \multirow[b]{2}{*}{$\mathrm{PPT}^{d}$} & \multicolumn{3}{|c|}{ Burned $^{e}$} & \multicolumn{3}{|c|}{ Burned $\times$ Year $f$} & \multirow[b]{2}{*}{ FRI $g$} & \multirow[b]{2}{*}{$\begin{array}{l}\text { FRI } \\
\text { trend }^{h}\end{array}$} \\
\hline & & & $\begin{array}{l}\text { Coef- } \\
\text { ficient }\end{array}$ & SE & $p$ & $\begin{array}{l}\text { Coeffi- } \\
\text { cient }\end{array}$ & SE & $p$ & & \\
\hline spring/summer & 81 & PYP & 0.10 & 0.46 & 0.83 & 0.09 & 0.19 & 0.62 & 0.02 & 0.04 \\
\hline winter & 46 & PYP & 0.40 & 0.68 & 0.54 & 0.18 & 0.27 & 0.50 & 0.16 & 0.16 \\
\hline \multicolumn{11}{|l|}{ Mountain Quail (Oreortyx pictus; } \\
\hline \multirow{3}{*}{$\begin{array}{l}\text { MOUQ) } \\
\text { spring/summer } \\
\text { winter }\end{array}$} & & & & & & & & & & \\
\hline & 81 & TYP & 0.57 & 0.82 & 0.48 & -0.28 & 0.28 & 0.33 & 0.17 & -0.15 \\
\hline & 46 & PYP & 0.00 & 0.17 & 1.00 & 0.07 & 0.08 & 0.42 & -0.02 & 0.20 \\
\hline \multicolumn{11}{|l|}{$\begin{array}{l}\text { House Wren (Troglodytes aedon; } \\
\text { HOWR) }\end{array}$} \\
\hline spring/summer & 73 & CYP & 0.16 & 0.55 & 0.76 & 0.23 & 0.12 & 0.07 & 0.05 & 0.17 \\
\hline winter & 43 & PYP & 0.17 & 0.15 & 0.26 & 0.00 & 0.05 & 0.97 & 0.10 & 0.00 \\
\hline $\begin{array}{l}\text { Mountain Bluebird (Sialia currucoides; } \\
\text { MOBL): winter }\end{array}$ & 26 & PYP & 0.14 & 0.40 & 0.71 & -0.21 & 0.18 & 0.23 & 0.07 & -0.21 \\
\hline \multicolumn{11}{|l|}{ Loggerhead Shrike (Lanius } \\
\hline \multicolumn{11}{|l|}{ ludovicianus; LOSH) } \\
\hline $\begin{array}{l}\text { spring/summer } \\
\text { winter }\end{array}$ & 40 & PYP & -0.02 & 0.08 & 0.79 & -0.03 & 0.02 & 0.15 & -0.09 & -0.27 \\
\hline \multicolumn{11}{|l|}{$\begin{array}{l}\text { Cooper's Hawk (Accipiter cooperii; } \\
\text { COHA) }\end{array}$} \\
\hline \multirow{2}{*}{$\begin{array}{l}\text { spring/summer } \\
\text { winter }\end{array}$} & 61 & PYP & 0.01 & 0.02 & 0.35 & 0.00 & 0.01 & 0.87 & 0.21 & -0.05 \\
\hline & 43 & PYP & 0.00 & 0.01 & 0.74 & 0.00 & 0.01 & 0.64 & -0.09 & 0.15 \\
\hline $\begin{array}{l}\text { Turkey Vulture (Cathartes aura; } \\
\text { TUVU): spring/summer }\end{array}$ & 81 & CYP & 0.01 & 0.05 & 0.79 & 0.00 & 0.02 & 0.99 & 0.05 & 0.00 \\
\hline \multicolumn{11}{|l|}{$\begin{array}{l}\text { Yellow-rumped Warbler (Setophaga } \\
\text { coronata; YRWA) }\end{array}$} \\
\hline \multirow{2}{*}{$\begin{array}{l}\text { spring/summer } \\
\text { winter }\end{array}$} & 35 & CYP & 0.02 & 0.09 & 0.80 & -0.05 & 0.02 & 0.02 & 0.11 & -0.54 \\
\hline & 34 & PYP & -0.07 & 0.33 & 0.83 & -0.08 & 0.15 & 0.58 & -0.06 & -0.19 \\
\hline \multicolumn{11}{|l|}{$\begin{array}{l}\text { California Quail (Callipepla } \\
\text { californica; CAQU) }\end{array}$} \\
\hline spring/summer & 73 & PYP & 1.22 & 1.33 & 0.34 & 1.07 & 0.44 & 0.01 & 0.17 & 0.37 \\
\hline winter & 43 & PYP & -0.60 & 1.08 & 0.56 & 0.06 & 0.43 & 0.88 & -0.13 & 0.03 \\
\hline \multicolumn{11}{|l|}{ Anna's Hummingbird (Calypte anna; } \\
\hline \multirow{2}{*}{$\begin{array}{l}\text { spring/summer } \\
\text { winter }\end{array}$} & 81 & CYP & 0.12 & 0.16 & 0.44 & -0.04 & 0.06 & 0.57 & 0.13 & -0.07 \\
\hline & 42 & PYP & -0.01 & 0.02 & 0.59 & 0.00 & 0.01 & 0.95 & -0.10 & 0.02 \\
\hline \multicolumn{11}{|l|}{ Phainopepla (Phainopepla nitens; } \\
\hline spring/summer & 76 & PYP & 0.14 & 0.18 & 0.41 & 0.21 & 0.07 & 0.00 & 0.09 & 0.39 \\
\hline winter & 41 & PYP & -0.03 & 0.14 & 0.82 & -0.04 & 0.04 & 0.23 & -0.06 & -0.30 \\
\hline $\begin{array}{l}\text { Ash-throated Flycatcher (Myiarchus } \\
\text { cinerascens; ATFL): spring/summer }\end{array}$ & 81 & PYP & -0.03 & 0.24 & 0.89 & -0.07 & 0.07 & 0.32 & -0.01 & -0.04 \\
\hline $\begin{array}{l}\text { Northern Mockingbird (Mimus } \\
\text { polyglottos; NOMO) }\end{array}$ & & & & & & & & & & \\
\hline spring/summer & 67 & CYP & 0.02 & 0.13 & 0.85 & -0.10 & 0.03 & 0.00 & 0.09 & -0.39 \\
\hline winter & 33 & PYP & -0.01 & 0.03 & 0.81 & 0.02 & 0.01 & 0.26 & -0.10 & 0.26 \\
\hline $\begin{array}{l}\text { Dark-eyed Junco (Junco hyemalis; } \\
\text { DEJU) }\end{array}$ & & & & & & & & & & \\
\hline spring/summer & 53 & PYP & -0.15 & 0.36 & 0.66 & -0.10 & 0.10 & 0.31 & -0.05 & -0.07 \\
\hline winter & 43 & PYP & 0.55 & 2.79 & 0.84 & 0.91 & 0.74 & 0.22 & 0.03 & 0.14 \\
\hline $\begin{array}{l}\text { Lark Sparrow (Chondestes grammacus; } \\
\text { LASP) }\end{array}$ & & & & & & & & & & \\
\hline spring/summer & 81 & PYP & 0.15 & 0.26 & 0.57 & -0.02 & 0.08 & 0.85 & 0.11 & -0.02 \\
\hline winter & 36 & PYP & -0.11 & 0.40 & 0.78 & 0.13 & 0.17 & 0.44 & -0.17 & 0.33 \\
\hline $\begin{array}{l}\text { Common Raven (Corvus corax; } \\
\text { CORA) }\end{array}$ & & & & & & & & & & \\
\hline spring/summer & 81 & PYP & -0.02 & 0.17 & 0.89 & -0.05 & 0.07 & 0.51 & -0.01 & -0.05 \\
\hline winter & 46 & PYP & -0.14 & 0.40 & 0.70 & 0.00 & 0.16 & 0.99 & -0.05 & 0.00 \\
\hline
\end{tabular}




\begin{tabular}{|c|c|c|c|c|c|c|c|c|c|c|}
\hline \multirow[b]{2}{*}{ Species and season ${ }^{b}$} & \multirow[b]{2}{*}{ Routes $^{c}$} & \multirow[b]{2}{*}{$\mathrm{PPT}^{d}$} & \multicolumn{3}{|c|}{ Burned $^{e}$} & \multicolumn{3}{|c|}{ Burned $\times$ Year $f$} & \multirow[b]{2}{*}{$\mathrm{FRI}^{g}$} & \multirow[b]{2}{*}{$\begin{array}{l}\text { FRI } \\
\text { trend }^{h}\end{array}$} \\
\hline & & & $\begin{array}{l}\text { Coef- } \\
\text { ficient }\end{array}$ & SE & $p$ & $\begin{array}{l}\text { Coeffi- } \\
\text { cient }\end{array}$ & SE & $p$ & & \\
\hline $\begin{array}{l}\text { Bullock's Oriole (Icterus bullockii; } \\
\text { BUOR): spring/summer }\end{array}$ & 68 & PYP & -0.01 & 0.13 & 0.91 & 0.04 & 0.04 & 0.32 & -0.03 & 0.14 \\
\hline \multicolumn{11}{|l|}{$\begin{array}{l}\text { Red-shouldered Hawk (Buteo lineatus; } \\
\text { RSHA) }\end{array}$} \\
\hline spring/summer & 58 & PYP & 0.02 & 0.05 & 0.66 & 0.02 & 0.01 & 0.13 & 0.19 & 0.38 \\
\hline winter & 40 & PYP & -0.05 & 0.06 & 0.32 & 0.01 & 0.02 & 0.49 & -0.29 & 0.12 \\
\hline \multicolumn{11}{|l|}{$\begin{array}{l}\text { Spotted Towhee (Pipilo maculatus; } \\
\text { SPTO) }\end{array}$} \\
\hline spring/summer & 81 & PYP & -0.25 & 0.39 & 0.51 & 0.70 & 0.12 & 0.00 & -0.06 & 0.24 \\
\hline winter & 46 & PYP & -0.20 & 0.51 & 0.69 & 0.26 & 0.14 & 0.07 & -0.11 & 0.21 \\
\hline \multicolumn{11}{|l|}{ California Towhee (Melozone crissalis; } \\
\hline $\begin{array}{l}\text { CALT) } \\
\text { spring/summer }\end{array}$ & 81 & PYP & -0.49 & 0.27 & 0.07 & 0.34 & 0.08 & 0.00 & -0.16 & 0.19 \\
\hline winter & 46 & PYP & -0.06 & 0.28 & 0.82 & 0.21 & 0.08 & 0.01 & -0.05 & 0.20 \\
\hline \multicolumn{11}{|l|}{$\begin{array}{l}\text { Western Bluebird (Sialia mexicana; } \\
\text { WEBL) }\end{array}$} \\
\hline spring/summer & 73 & PYP & -0.37 & 0.32 & 0.24 & -0.19 & 0.10 & 0.05 & -0.16 & -0.18 \\
\hline winter & 43 & PYP & -0.19 & 0.71 & 0.78 & -0.21 & 0.24 & 0.37 & -0.05 & -0.15 \\
\hline $\begin{array}{l}\text { Warbling Vireo (Vireo gilvus; WAVI): } \\
\text { spring/summer }\end{array}$ & 65 & CYP & -0.03 & 0.06 & 0.64 & -0.01 & 0.03 & 0.69 & -0.11 & -0.12 \\
\hline \multicolumn{11}{|l|}{$\begin{array}{l}\text { American Crow (Corvus } \\
\text { brachyrhynchos; AMCR) }\end{array}$} \\
\hline spring/summer & 70 & CYP & -0.08 & 0.22 & 0.71 & -0.09 & 0.06 & 0.09 & -0.07 & -0.21 \\
\hline winter & 41 & PYP & -0.24 & 0.56 & 0.66 & 0.09 & 0.15 & 0.56 & -0.16 & 0.11 \\
\hline $\begin{array}{l}\text { White-crowned Sparrow (Zonotrichia } \\
\text { leucophrys; WCSP): winter }\end{array}$ & 46 & PYP & -1.48 & 2.37 & 0.51 & 2.42 & 0.82 & 0.00 & -0.13 & 0.30 \\
\hline $\begin{array}{l}\text { Rufous-crowned Sparrow (Aimophila } \\
\text { ruficeps; RCSP): spring/summer }\end{array}$ & 42 & CYP & -0.06 & 0.23 & 0.76 & 0.18 & 0.05 & 0.00 & -0.14 & 0.46 \\
\hline $\begin{array}{l}\text { Vesper Sparrow (Pooecetes gramineus; } \\
\text { VESP): winter }\end{array}$ & 30 & PYP & -0.05 & 0.09 & 0.56 & -0.06 & 0.05 & 0.26 & -0.15 & -0.41 \\
\hline $\begin{array}{l}\text { Sharp-shinned Hawk (Accipiter } \\
\text { striatus; SSHA): winter }\end{array}$ & 44 & PYP & -0.01 & 0.02 & 0.68 & 0.01 & 0.01 & 0.39 & -0.16 & 0.28 \\
\hline \multicolumn{11}{|l|}{$\begin{array}{l}\text { Band-tailed Pigeon (Patagioenas } \\
\text { fasciata: BTPI) }\end{array}$} \\
\hline spring/summer & 67 & PYP & -0.28 & 0.24 & 0.22 & -0.43 & 0.09 & 0.00 & -0.11 & -0.43 \\
\hline winter & 37 & PYP & -1.74 & 0.92 & 0.05 & -0.82 & 0.29 & 0.00 & -0.22 & -0.22 \\
\hline $\begin{array}{l}\text { Golden-crowned Sparrow (Zonotrichia } \\
\text { atricapilla; GCSP): winter }\end{array}$ & 46 & PYP & -0.25 & 0.20 & 0.19 & -0.21 & 0.09 & 0.01 & -0.17 & -0.37 \\
\hline $\begin{array}{l}\text { Western Flycatcher (Empidonax } \\
\text { difficilis; PSFL): spring/summer }\end{array}$ & 73 & CYP & -0.04 & 0.06 & 0.41 & -0.03 & 0.02 & 0.15 & -0.18 & -0.33 \\
\hline \multicolumn{11}{|l|}{ Brown Creeper (Certhia americana; } \\
\hline spring/summer & 21 & PYP & -0.07 & 0.10 & 0.44 & 0.00 & 0.03 & 0.97 & -0.15 & 0.00 \\
\hline winter & 10 & PYP & -0.08 & 0.05 & 0.05 & 0.05 & 0.03 & 0.09 & -0.21 & 0.24 \\
\hline $\begin{array}{l}\text { Brewer's Blackbird (Euphagus } \\
\text { cyanocephalus; BRBL): spring/ } \\
\text { summer }\end{array}$ & 63 & CYP & -0.06 & 0.11 & 0.59 & 0.01 & 0.06 & 0.82 & -0.18 & 0.09 \\
\hline \multicolumn{11}{|l|}{$\begin{array}{l}\text { Nuttall's Woodpecker (Picoides } \\
\text { nuttallii; NUWO) }\end{array}$} \\
\hline spring/summer & 73 & PYP & -0.29 & 0.14 & 0.04 & -0.06 & 0.03 & 0.05 & -0.31 & -0.13 \\
\hline winter & 43 & PYP & -0.06 & 0.15 & 0.71 & -0.01 & 0.03 & 0.76 & -0.08 & -0.03 \\
\hline $\begin{array}{l}\text { Brown-headed Cowbird (Molothrus } \\
\text { ater; BHCO): spring/summer }\end{array}$ & 79 & PYP & -0.05 & 0.05 & 0.26 & -0.01 & 0.01 & 0.55 & -0.20 & -0.07 \\
\hline $\begin{array}{l}\text { Hermit Thrush (Catharus guttatus; } \\
\text { HETH): winter }\end{array}$ & 34 & PYP & -0.07 & 0.14 & 0.57 & -0.04 & 0.04 & 0.31 & -0.20 & -0.26 \\
\hline
\end{tabular}


TABLE 3 (continued).

\begin{tabular}{|c|c|c|c|c|c|c|c|c|c|c|}
\hline \multirow[b]{2}{*}{ Species and season ${ }^{b}$} & \multirow[b]{2}{*}{ Routes $^{c}$} & \multirow[b]{2}{*}{$\mathrm{PPT}^{d}$} & \multicolumn{3}{|c|}{ Burned $^{e}$} & \multicolumn{3}{|c|}{ Burned $\times$ Year $f$} & \multirow[b]{2}{*}{ FRI $g$} & \multirow[b]{2}{*}{$\begin{array}{l}\text { FRI } \\
\text { trend }^{h}\end{array}$} \\
\hline & & & $\begin{array}{l}\text { Coef- } \\
\text { ficient }\end{array}$ & SE & $p$ & $\begin{array}{l}\text { Coeffi- } \\
\text { cient }\end{array}$ & SE & $p$ & & \\
\hline $\begin{array}{l}\text { Western Wood Pewee (Contopus } \\
\text { sordidulus; WEWP): spring/summer }\end{array}$ & 73 & PYP & -0.21 & 0.18 & 0.22 & -0.05 & 0.04 & 0.21 & -0.21 & -0.11 \\
\hline \multicolumn{11}{|l|}{$\begin{array}{l}\text { California Scrub-Jay (Aphelocoma } \\
\text { californica; WESJ) }\end{array}$} \\
\hline spring/summer & 81 & PYP & -1.40 & 0.45 & 0.00 & 0.14 & 0.12 & 0.25 & -0.25 & 0.05 \\
\hline winter & 46 & PYP & -0.93 & 0.96 & 0.31 & 0.13 & 0.21 & 0.54 & -0.18 & 0.05 \\
\hline \multicolumn{11}{|l|}{$\begin{array}{l}\text { Hairy Woodpecker (Picoides villosus; } \\
\text { HAWO) }\end{array}$} \\
\hline spring/summer & 38 & PYP & -0.21 & 0.10 & 0.02 & 0.08 & 0.04 & 0.03 & -0.26 & 0.20 \\
\hline winter & 25 & PYP & -0.12 & 0.12 & 0.28 & -0.03 & 0.04 & 0.41 & -0.23 & -0.15 \\
\hline $\begin{array}{l}\text { Greater Roadrunner (Geococcyx } \\
\text { californianus; GRRO): spring/ } \\
\text { summer }\end{array}$ & 50 & PYP & -0.09 & 0.04 & 0.03 & -0.05 & 0.02 & 0.00 & -0.25 & -0.32 \\
\hline \multicolumn{11}{|l|}{$\begin{array}{l}\text { Wild Turkey (Meleagris gallopavo; } \\
\text { WITU) }\end{array}$} \\
\hline spring/summer & 58 & PYP & -0.02 & 0.24 & 0.91 & -0.03 & 0.08 & 0.75 & -0.02 & -0.04 \\
\hline winter & 33 & PYP & -0.99 & 0.52 & 0.05 & -0.46 & 0.20 & 0.02 & -0.48 & -0.48 \\
\hline $\begin{array}{l}\text { Brewer's Sparrow (Spizella breweri; } \\
\text { BRSP): spring/summer }\end{array}$ & 47 & PYP & -0.09 & 0.07 & 0.20 & 0.13 & 0.05 & 0.02 & -0.27 & 0.54 \\
\hline \multicolumn{11}{|l|}{$\begin{array}{l}\text { Northern Flicker (Colaptes auratus; } \\
\text { NOFL) }\end{array}$} \\
\hline spring/summer & 73 & PYP & -0.21 & 0.09 & 0.03 & -0.05 & 0.02 & 0.03 & -0.37 & -0.17 \\
\hline winter & 43 & PYP & -0.13 & 0.12 & 0.23 & -0.06 & 0.03 & 0.08 & -0.22 & -0.19 \\
\hline \multicolumn{11}{|l|}{$\begin{array}{l}\text { Ladder-backed Woodpecker (Picoides } \\
\text { scalaris; LBWO) }\end{array}$} \\
\hline spring/summer & 34 & PYP & -0.04 & 0.04 & 0.28 & -0.03 & 0.02 & 0.10 & -0.10 & -0.20 \\
\hline winter & 24 & PYP & -0.49 & 0.10 & 0.00 & -0.14 & 0.03 & 0.00 & -0.49 & -0.31 \\
\hline $\begin{array}{l}\text { Savannah Sparrow (Passerculus } \\
\text { sandwichensis; SAVS): winter }\end{array}$ & 31 & PYP & -0.72 & 0.71 & 0.28 & -0.67 & 0.27 & 0.01 & -0.31 & -0.61 \\
\hline \multicolumn{11}{|l|}{$\begin{array}{l}\text { American Robin (Turdus migratorius; } \\
\text { AMRO) }\end{array}$} \\
\hline spring/summer & 61 & PYP & -0.15 & 0.07 & 0.04 & -0.03 & 0.02 & 0.11 & -0.63 & -0.23 \\
\hline winter & 37 & PYP & 0.00 & 0.07 & 0.99 & -0.01 & 0.04 & 0.87 & 0.00 & -0.06 \\
\hline $\begin{array}{l}\text { Black-headed Grosbeak (Pheucticus } \\
\text { melanocephalus; BHGR): spring/ } \\
\text { summer }\end{array}$ & 81 & CYP & -0.64 & 0.25 & 0.01 & -0.13 & 0.06 & 0.02 & -0.33 & -0.14 \\
\hline \multicolumn{11}{|l|}{$\begin{array}{l}\text { Oak Titmouse (Baeolophus inornatus; } \\
\text { OATI) }\end{array}$} \\
\hline spring/summer & 81 & TYP & -0.91 & 0.42 & 0.03 & 0.40 & 0.11 & 0.00 & -0.38 & 0.31 \\
\hline winter & 46 & PYP & -0.67 & 0.64 & 0.27 & 0.35 & 0.12 & 0.00 & -0.32 & 0.29 \\
\hline \multicolumn{11}{|l|}{$\begin{array}{l}\text { Purple Finch (Haemorhous purpureus; } \\
\text { PUFI) }\end{array}$} \\
\hline spring/summer & 45 & CYP & -0.08 & 0.12 & 0.49 & 0.00 & 0.04 & 0.91 & -0.17 & -0.02 \\
\hline winter & 32 & PYP & -0.10 & 0.06 & 0.09 & 0.01 & 0.03 & 0.70 & -0.54 & 0.12 \\
\hline $\begin{array}{l}\text { Horned Lark (Eremophila alpestris; } \\
\text { HOLA): spring/summer }\end{array}$ & 40 & CYP & -0.36 & 0.49 & 0.43 & 0.21 & 0.09 & 0.02 & -0.36 & 0.35 \\
\hline \multicolumn{11}{|l|}{$\begin{array}{l}\text { Orange-crowned Warbler (Oreothlypis } \\
\text { celata; OCWA) }\end{array}$} \\
\hline spring/summer & 77 & CYP & -0.07 & 0.05 & 0.15 & -0.04 & 0.02 & 0.03 & -0.31 & -0.40 \\
\hline winter & 40 & PYP & -0.04 & 0.03 & 0.14 & -0.01 & 0.01 & 0.28 & -0.41 & -0.36 \\
\hline $\begin{array}{l}\text { Violet-green Swallow (Tachycineta } \\
\text { thalassina; VGSW): spring/summer }\end{array}$ & 45 & PYP & -1.09 & 0.40 & 0.01 & -0.25 & 0.12 & 0.04 & -0.36 & -0.17 \\
\hline \multicolumn{11}{|l|}{$\begin{array}{l}\text { Western Meadowlark (Sturnella } \\
\text { neglecta; WEME) }\end{array}$} \\
\hline spring/summer & 49 & TYP & -0.17 & 0.41 & 0.67 & 0.02 & 0.07 & 0.78 & -0.17 & 0.04 \\
\hline winter & 35 & PYP & -0.83 & 0.38 & 0.03 & -0.18 & 0.16 & 0.25 & -0.55 & -0.24 \\
\hline
\end{tabular}




\begin{tabular}{|c|c|c|c|c|c|c|c|c|c|c|}
\hline \multirow[b]{2}{*}{ Species and season ${ }^{b}$} & \multirow[b]{2}{*}{ Routes $^{c}$} & \multirow[b]{2}{*}{$\mathrm{PPT}^{d}$} & \multicolumn{3}{|c|}{ Burned $^{e}$} & \multicolumn{3}{|c|}{ Burned $\times$ Year $f$} & \multirow[b]{2}{*}{ FRI ${ }^{g}$} & \multirow[b]{2}{*}{$\begin{array}{r}\text { FRI } \\
\text { trend }\end{array}$} \\
\hline & & & $\begin{array}{l}\text { Coef- } \\
\text { ficient }\end{array}$ & SE & $p$ & $\begin{array}{c}\text { Coeffi- } \\
\text { cient }\end{array}$ & SE & $p$ & & \\
\hline $\begin{array}{l}\text { Gray Vireo (Vireo vicinior; GRVI): } \\
\text { spring/summer }\end{array}$ & 45 & TYP & -0.16 & 0.22 & 0.44 & 0.07 & 0.03 & 0.01 & -0.37 & 0.30 \\
\hline \multicolumn{11}{|l|}{ Bewick's Wren (Thryomanes bewickii; } \\
\hline spring/summer & 81 & TYP & -1.59 & 0.25 & 0.00 & 0.41 & 0.06 & 0.00 & -0.55 & 0.27 \\
\hline winter & 46 & PYP & -0.24 & 0.19 & 0.19 & 0.17 & 0.05 & 0.00 & -0.21 & 0.26 \\
\hline \multicolumn{11}{|l|}{$\begin{array}{l}\text { Black-throated Sparrow (Amphispiza } \\
\text { bilineata; BTSP) }\end{array}$} \\
\hline spring/summer & 56 & PYP & -0.78 & 0.24 & 0.00 & -0.06 & 0.07 & 0.44 & -0.50 & -0.07 \\
\hline winter & 18 & PYP & -0.98 & 0.44 & 0.02 & -0.01 & 0.09 & 0.90 & -0.27 & -0.01 \\
\hline $\begin{array}{l}\text { Ruby-crowned Kinglet (Regulus } \\
\text { calendula; RCKI): winter }\end{array}$ & 46 & PYP & -0.58 & 0.24 & 0.01 & 0.05 & 0.07 & 0.49 & -0.42 & 0.07 \\
\hline $\begin{array}{l}\text { Purple Martin (Progne subis; PUMA): } \\
\text { spring/summer }\end{array}$ & 34 & TYP & -0.25 & 0.11 & 0.02 & 0.06 & 0.03 & 0.04 & -0.42 & 0.21 \\
\hline $\begin{array}{l}\text { Scott's Oriole (Icterus parisorum; } \\
\text { SCOR): spring/summer }\end{array}$ & 60 & PYP & -0.19 & 0.07 & 0.00 & 0.00 & 0.02 & 0.88 & -0.44 & 0.01 \\
\hline \multicolumn{11}{|l|}{$\begin{array}{l}\text { White-breasted Nuthatch (Sitta } \\
\text { carolinensis: WBNU) }\end{array}$} \\
\hline spring/summer & 69 & PYP & -0.45 & 0.18 & 0.01 & -0.11 & 0.05 & 0.02 & -0.43 & -0.21 \\
\hline winter & 42 & PYP & -0.63 & 0.24 & 0.01 & -0.07 & 0.05 & 0.13 & -0.50 & -0.12 \\
\hline $\begin{array}{l}\text { Western Tanager (Piranga ludoviciana; } \\
\text { WETA): spring/summer }\end{array}$ & 55 & PYP & -0.34 & 0.16 & 0.03 & -0.08 & 0.05 & 0.12 & -0.48 & -0.22 \\
\hline \multicolumn{11}{|l|}{$\begin{array}{l}\text { Acorn Woodpecker (Melanerpes } \\
\text { formicivorus; ACWO) }\end{array}$} \\
\hline spring/summer & 72 & PYP & -1.46 & 0.60 & 0.01 & -0.14 & 0.11 & 0.17 & -0.38 & -0.08 \\
\hline winter & 41 & PYP & -3.11 & 1.11 & 0.00 & -0.08 & 0.15 & 0.59 & -0.59 & -0.03 \\
\hline \multicolumn{11}{|l|}{ Steller's Jay (Cyanocitta stelleri; STJA) } \\
\hline $\begin{array}{l}\text { spring/summer } \\
\text { winter }\end{array}$ & 52 & CYP & -0.68 & 0.40 & 0.08 & 0.07 & 0.08 & 0.36 & -0.32 & 0.07 \\
\hline winter & 29 & PYP & -2.67 & 0.59 & 0.00 & 0.10 & 0.14 & 0.48 & -0.70 & 0.05 \\
\hline $\begin{array}{l}\text { Fox Sparrow (Passerella iliaca; FOSP): } \\
\text { winter }\end{array}$ & 46 & PYP & -1.49 & 0.80 & 0.06 & 0.27 & 0.10 & 0.01 & -0.51 & 0.18 \\
\hline \multicolumn{11}{|l|}{ Bushtit (Psaltriparus minimus; BUSH) } \\
\hline $\begin{array}{l}\text { spring/summer } \\
\text { winter }\end{array}$ & 46 & PYP & -1.57 & 0.36 & 0.00 & 0.12 & 0.19 & 0.57 & $\begin{array}{l}-0.40 \\
-0.60\end{array}$ & $\begin{array}{l}0.20 \\
0.09\end{array}$ \\
\hline $\begin{array}{l}\text { Olive-sided Flycatcher (Contopus } \\
\text { cooperi; OSFL): spring/summer }\end{array}$ & 46 & TYP & -0.10 & 0.03 & 0.00 & -0.03 & 0.01 & 0.03 & -0.59 & -0.36 \\
\hline
\end{tabular}

("Year," values 1-5). Other covariate fixed effects were date, precipitation, elevation, and pre-fire abundance on the basis of atlas data ("Atlas abundance," defined below). The route was included as a random-effect variable in each model (with varying intercept). This helped control for any pre-existing differences among routes not already controlled for by elevation and pre-fire abundance, and also controlled for differences among observers, since observers were assigned to routes. Thus, the full additive model for each species' abundance $(Y)$ was

$$
\begin{gathered}
Y=\text { Burned }+ \text { Year }+ \text { Date }+ \text { Precipitation }+ \\
\text { Elevation }+ \text { Atlas abundance }+(1 \mid \text { Route })+\varepsilon
\end{gathered}
$$

where 1| indicates random intercept, and $\varepsilon$ represents an error term for additional variation not explained by the model.

We assigned each unburned control route the same value for year since fire as that of its most comparable burned route. Thus the interaction between Burned and Year represents the postfire trend on burned routes relative to that on unburned control routes. Survey date was standardized separately for spring and winter, with the earliest survey date set to 1 (up to 122 in spring and to 103 in winter). We based precipitation on average cumulative annual rainfall from July to June at five weather stations in the region (each within $3.2 \mathrm{~km}$ of a study route and equal in average elevation to the average elevation of study routes, $\sim 1280 \mathrm{~m}$ ): Cuyamaca (www.wrcc.dri.edu, 
TABLE 3 (continued).

\begin{tabular}{|c|c|c|c|c|c|c|c|c|c|c|}
\hline \multirow[b]{2}{*}{ Species and season ${ }^{b}$} & \multirow[b]{2}{*}{ Routes $^{c}$} & \multirow[b]{2}{*}{$\mathrm{PPT}^{d}$} & \multicolumn{3}{|c|}{ Burned $^{e}$} & \multicolumn{3}{|c|}{ Burned $\times$ Year $f$} & \multirow[b]{2}{*}{ FRI $g$} & \multirow[b]{2}{*}{$\begin{array}{l}\text { FRI } \\
\text { trend }^{h}\end{array}$} \\
\hline & & & $\begin{array}{l}\text { Coef- } \\
\text { ficient }\end{array}$ & SE & $p$ & $\begin{array}{c}\text { Coeffi- } \\
\text { cient }\end{array}$ & SE & $p$ & & \\
\hline \multicolumn{11}{|l|}{$\begin{array}{l}\text { California Thrasher (Toxostoma } \\
\text { redivivum: } \text { CATH) }\end{array}$} \\
\hline spring/summer & 81 & CYP & -0.73 & 0.11 & 0.00 & 0.09 & 0.03 & 0.00 & -0.63 & 0.15 \\
\hline winter & 46 & PYP & -0.49 & 0.12 & 0.00 & 0.11 & 0.04 & 0.00 & -0.59 & 0.26 \\
\hline $\begin{array}{l}\text { Cassin’s Vireo (Vireo cassiniz; CAVI): } \\
\text { spring/summer }\end{array}$ & 27 & CYP & -0.07 & 0.04 & 0.08 & 0.01 & 0.02 & 0.46 & -0.62 & 0.21 \\
\hline $\begin{array}{l}\text { Hutton's Vireo (Vireo huttoni; HUVI): } \\
\text { spring/summer }\end{array}$ & 65 & CYP & -0.04 & 0.02 & 0.01 & 0.00 & 0.01 & 0.68 & -0.62 & -0.08 \\
\hline \multicolumn{11}{|l|}{$\begin{array}{l}\text { Pygmy Nuthatch (Sitta pygmaea; } \\
\text { PYNU) }\end{array}$} \\
\hline spring/summer & 26 & CYP & -0.87 & 0.29 & 0.00 & -0.27 & 0.11 & 0.01 & -0.55 & -0.35 \\
\hline winter & 18 & PYP & -1.27 & 0.38 & 0.00 & -0.44 & 0.12 & 0.00 & -0.81 & -0.61 \\
\hline \multicolumn{10}{|l|}{ Wrentit (Chamaea fasciata; WREN) } & 0.28 \\
\hline & 46 & PYP & -0.72 & 0.22 & 0.00 & 0.08 & 0.05 & 0.08 & -0.62 & 0.14 \\
\hline $\begin{array}{l}\text { Cactus Wren (Campylorbynchus } \\
\text { brunneicapillus; CACW): spring/ } \\
\text { summer }\end{array}$ & 34 & CYP & -0.17 & 0.05 & 0.00 & -0.01 & 0.02 & 0.66 & -0.70 & -0.09 \\
\hline $\begin{array}{l}\text { Mountain Chickadee (Poecile gambeli; } \\
\text { MOCH) }\end{array}$ & & & & & & & & & & \\
\hline $\begin{array}{l}\text { spring/summer } \\
\text { winter }\end{array}$ & 48 & PYP & -1.89 & 0.45 & 0.00 & 0.20 & 0.09 & 0.03 & -0.71 & 0.15 \\
\hline winter & 31 & PYP & -2.42 & 0.52 & 0.00 & 0.21 & 0.13 & 0.11 & -0.74 & 0.12 \\
\hline $\begin{array}{l}\text { Blue-gray Gnatcatcher (Polioptila } \\
\text { caerulea; BGGN): spring/summer }\end{array}$ & 77 & CYP & -0.35 & 0.08 & 0.00 & 0.07 & 0.02 & 0.00 & -0.78 & 0.30 \\
\hline
\end{tabular}

${ }^{a}$ Species ordered by Fire Response Index (see text for calculation; ordered by average of spring/summer and winter if species occurs during both seasons. Gray highlighting indicates a relatively neutral response (Fire Response Index -0.10 to +0.10 .).

${ }^{b}$ Spring-sum-mer, 15 April-15 July; winter, 1 December-29 February.

${ }^{c}$ Number of qualifying routes (out of 81 possible in spring/summer and 46 possible in winter).

${ }^{d}$ Season of precipitation with which the species' post-fire pattern of change in spring/summer was most closely correlated. CYP, current year's precipitation (July-June, including the April-July surveys of that year); PYP, previous year's precipitation; TYP, current year's and previous year's precipitation combined. Only PYP was used in winter.

${ }^{e}$ Coefficient of contribution of fire to post-fire abundance, unstandardized (i.e., the difference in number of birds per km on burned routes relative to unburned routes). Statistically significant values $(p<0.05)$ are in boldface for species with FRI $\geq 0.10$ or $\leq-0.10$. $f$ Coefficient of interactive effect of fire and year since fire, unstandardized. Statistically significant values $(p<0.05)$ are in boldface. ${ }^{g}$ Fire Response Index.

${ }^{5}$-year trend in Fire Response Index.

accessed 31 Jan 2013), Oak Grove, Descanso, Ranchita, and Mount Laguna (http://www.raws. dri.edu, accessed 4 Feb 2013). For winter, we used the previous year's precipitation (PYP). For example, for surveys from December 2004 to February 2005 , PYP is based on precipitation from July 2003 to June 2004. For spring, for each species we used one of three precipitation variables that most positively correlated with abundance of that species (previous year's precipitation or PYP, current year's precipitation or CYP, or these 2 years' precipitation combined or TYP). For example, for surveys from April to June 2005, PYP is based on precipitation from July 2003 to June 2004. We defined each route's elevation as the average of its start and end points. For species along each route, we defined Atlas abundance as the species' average pre-fire abundance in the square (number of birds per hour), excluding surveys under 1 hour and any nocturnal surveys. The variables Date, Precipitation, Elevation, and Atlas abundance were standardized and centered for each species and season (mean $=0, S D=1$ ).

We evaluated the significance of Burned by a likelihood-ratio test of the full additive model with the effect in question against the model without the effect in question. Likewise, we evaluated the significance of the interaction of Burned and Year by a likelihood-ratio test of the full additive model against the same model with the interaction included. We used the models' predictions to calculate, for each species, the average proportion 
("Fire Response Index") of birds/km in burned habitat relative to unburned over the entire 5 years post-fire pooled: $(B-U) /(B+U)$, where $B$ is the number per $\mathrm{km}$ predicted by the model along burned routes, averaged over the 5 years, and $U$ is the number per kilometer predicted by the model along unburned routes, also averaged over the 5 years. Similarly, we calculated the "Trend in Fire Response Index" over 5 years as $\left[\left(B_{5}-B_{1}\right)-\left(U_{5}\right.\right.$ $\left.\left.-U_{1}\right)\right] /\left(B_{5}+B_{1}+U_{5}+U_{1}\right)$.

\section{COMPARISON OF ATLAS SQUARES AND RECOVERY ON BURNED ROUTES}

We also present descriptive results for all species with at least 5 records either pre-fire or post-fire, including those with data insufficient for modeling. For each species meeting this criterion, we counted the total number of atlas squares with burned routes on which the species was present pre-fire vs. post-fire. We also calculated yearto-year change as the proportional difference in abundance on burned routes sampled in consecutive years post-fire, as $\left(Y_{n}-Y_{n-1}\right) /\left(Y_{n}+Y_{n-1}\right)$, where $Y$ is the abundance (birds $/ \mathrm{km}$ ) and $n$ is the year. Dividing by the sum makes these metrics a proportional change scaled from -100 to $+100 \%$ for all species and circumvents the division by zero in the cases of change from absence to presence. We also correlated annual year-to-year change with precipitation and elevation. Pearson correlation analysis was done in program $\mathrm{R}$, other descriptive statistics in Excel.

\section{RESULTS}

\section{Comparative Post-FIRE RESPONSES}

A total of 92 species qualified for post-fire modeling in spring/summer and/or winter (Table 3). In spring/summer, over the 5 years after the fires, 24 species of the 81 that qualified for modeling at this season responded positively to fire by the criterion Fire Response Index $\geq 0.10$, while 43 species responded negatively by the criterion Fire Response Index $\leq-0.10$. Of the 24 positive species, the response of 6 was statistically significant $(p<0.05)$ by the likelihood-ratio test of the variable Burned (Lazuli Bunting, Rock Wren, Costa’s Hummingbird, Black-chinned Sparrow, Redtailed Hawk, and Lesser Goldfinch). Of the negative species, the response of 25 was statistically significant according to this test, led by the Blue-gray Gnatcatcher, Mountain Chickadee, Cactus Wren, Wrentit, Hutton's Vireo, and California Thrasher. In winter, 8 species of the 60 that qualified for modeling at this season responded positively over the 5 years after the fires (Fire Response Index $\geq$ 0.10 ), while 34 species responded negatively (Fire Response Index $\leq-0.10)$. None of the positive responses in winter was statistically significant according to the likelihood-ratio test, but 15 negative responses were significant, led by those of the Pygmy Nuthatch, Mountain Chickadee, Steller's Jay, Wrentit, and Bushtit. Of the 49 species modeled in both seasons, in only 3 was the response positive in one season and negative in the other, and in none of these was a response statistically significant according to the likelihood-ratio test (Red-shouldered Hawk, California Quail, and Lark Sparrow). In many species, however, the strength of the response in the two seasons differed, with that of many positive responders being stronger in spring/summer than in winter (e.g., Rock Wren). Differences among negative responders were mixed, with some responses being more negative in spring/summer (e.g., Nuttall's Woodpecker), others in winter (e.g., Steller's Jay).

The strength of post-fire trends on burned routes relative to those on unburned controls is indicated by the interaction (Burned $\times$ Year) in the post-fire model. For 36 species in spring/ summer and 12 in winter, this interaction was statistically significant (Table 3). For positively responding species, a negative interaction may indicate a strong initial response on burned routes that subsequently lessens (e.g., Rock Wren and Lawrence's Goldfinch), or a positive interaction may indicate an initially weak response on burned routes that subsequently intensifies (e.g., Black-chinned Sparrow). For negatively responding species, a positive interaction may indicate recovery in burned areas (e.g., Oak Titmouse), and a negative interaction may indicate an initially neutral response with subsequent decline in burned areas relative to unburned (e.g., Band-tailed Pigeon). The trend on burned routes relative to that on unburned routes can also be expressed as a difference between year 1 and year 5 in the Fire Response Index, as predicted by the post-fire model. This approach allows for a more direct comparison among all species that fall on a spectrum of positive to negative responses and a spectrum of positive to negative trends (Figures 2 and 3). For example, in spring/summer (Figure 2), species that fall in the upper left quadrant are positive responders that decreased over time (e.g., Lazuli Bunting and Rock Wren), species in the upper right quadrant are positive responders that became increasingly positive over time (e.g., Dusky Flycatcher and White-throated Swift), species in the lower left quadrant are negative responders 


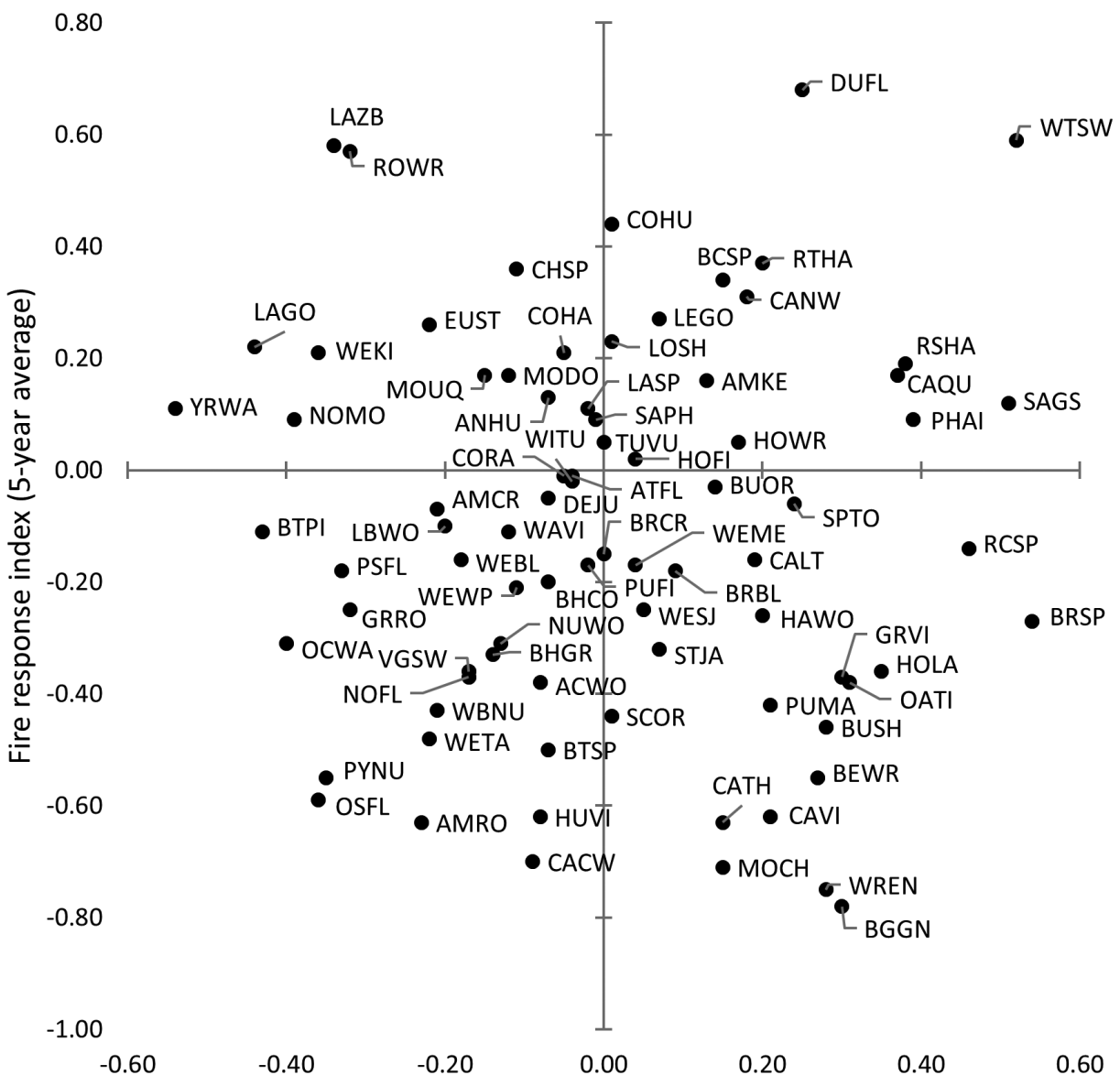

Five-year trend in fire response index

FIGURE 2. Comparison of species by overall response to fire ( $y$ axis) and 5-year trend ( $x$ axis) in spring/summer, 2003-2008. Species' codes are given in Table 3.

that became increasingly negative over time (e.g., Pygmy Nuthatch and Olive-sided Flycatcher), and species in the lower right quadrant are negative responders that recovered to some extent over time (e.g., Wrentit and Blue-gray Gnatcatcher). However, the increase on burned routes relative to unburned controls was sometimes driven more strongly by declines in unburned areas than by recovery, as was the case for the Gray Vireo and Purple Martin.

To explore post-fire trends further, we also modeled Year as a categorical variable, enabling comparison of year-to-year variation relative to linear trends. This method reveals additional complexity in each category of response, from species with neutral patterns (Figure 4) to those in which positive or negative trends contrast with positive or negative responses (Figures 5 and 6). It is also useful for comparing species by season, from those with little seasonal difference (Figure 7) to those with stronger differences (Figures 8-10), and for examining the interaction of Burned and Year, which reveals for the Spotted Towhee a reversal of trend from negative to positive over the 5 years (Figure 11).

\section{EFFECTS OF PRECIPITATION AND ELEVATION ON RECOVERY}

Post-fire models controlled for the effects of precipitation and elevation to isolate the main effects of fire and year on abundance. However, it was apparent that the trends of many species closely tracked the wide swings in precipitation in the years after the fires, strongly affecting recovery. To explore these effects further, we assessed the correlations of precipitation and elevation with year-to-year proportional changes in abundance.

Strong correlations of precipitation with 


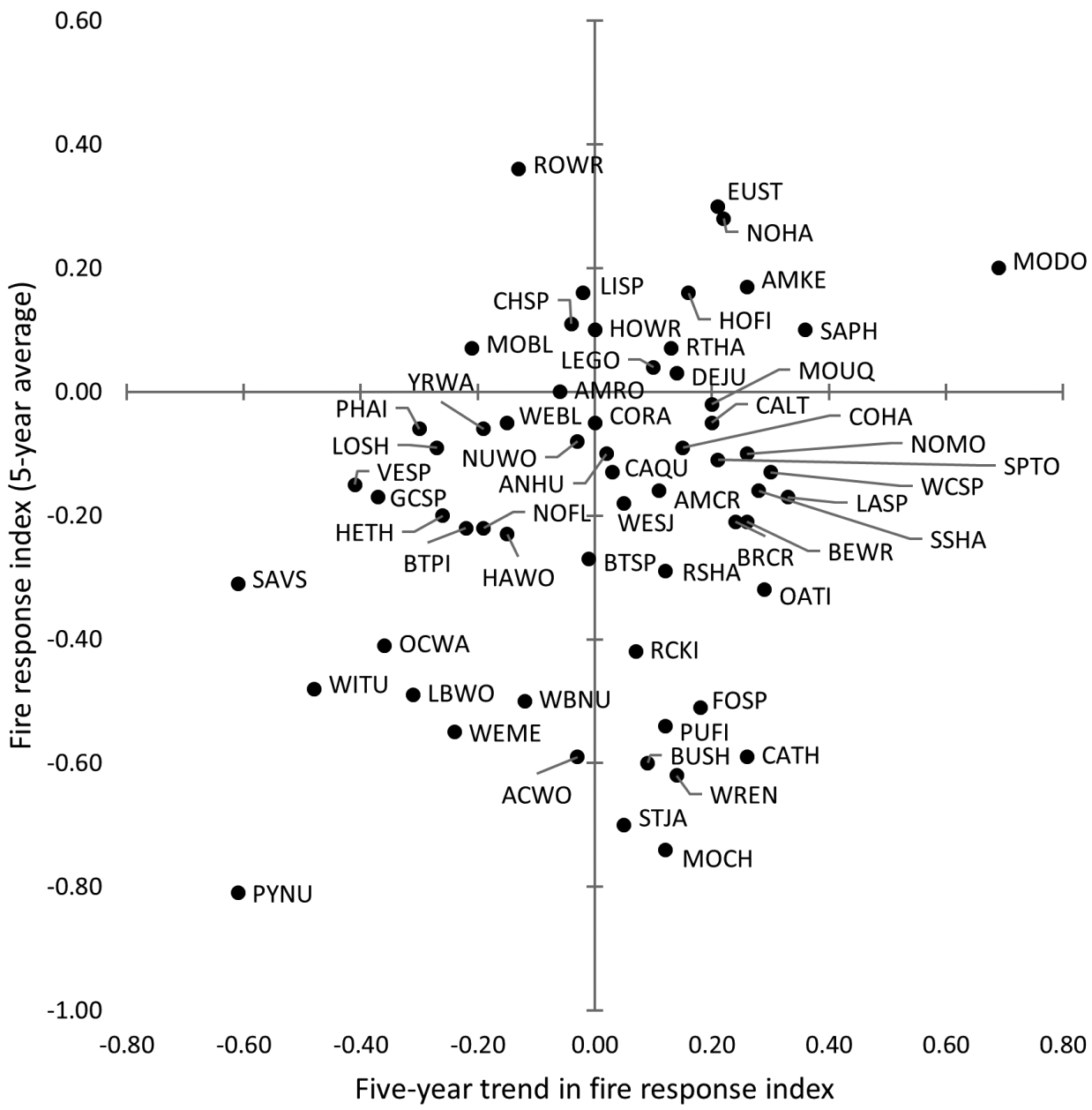

FIGURE 3. Comparison of species by overall response to fire ( $y$ axis) and 5-year trend ( $x$ axis) in winter, 2003-2008. Species' codes are given in Table 3.

year-to-year proportional changes in abundance were most apparent in winter, for some species occurring year round, such as the Lesser Goldfinch and House Wren, as well as for winter visitors such as the White-crowned Sparrow and Yellow-rumped Warbler. All of these increased in the year following an increase in rain in the previous year (PYP; July to June) (Figure 12). Other less numerous species that followed the same pattern were the Golden-crowned, Savannah, Chipping, Brewer's, and Lark sparrows. In spring/ summer, many migratory species, such as Costa's Hummingbird and the Lazuli Bunting, were more closely correlated with the current year's precipitation (CYP), while year-round residents were more likely to be correlated with the previous year's precipitation (e.g., Black-throated Sparrow), or with the current and previous years' precipitation combined (TYP; e.g., Bewick's Wren) (Figure 13). More species tended to be positively than negatively correlated with precipitation both in winter for PYP $\left(n=60, \chi^{2}=8.07, \mathrm{df}=1, p=0.004\right)$ and in spring/summer for the three categories of precipitation (PYP, CYP, and TYP) pooled $(n=$ 81 species $\times 3$ categories $=243, \chi^{2}=6.9, \mathrm{df}=1$, $p=0.01)$. In general, species that responded to fire positively tended to respond to precipitation more strongly than did those that responded to fire negatively, in both winter and spring/summer (Figure 14).

Year-to-year changes in abundance on burned routes also tended to be positively correlated with elevation, but, across species, more strongly so in spring/summer $\left(n=81, \chi^{2}=16.90, \mathrm{df}=1, p<\right.$ $0.001)$ than in winter $\left(n=60, \chi^{2}=0.50, \mathrm{df}=1\right.$, $p=0.44)$. 

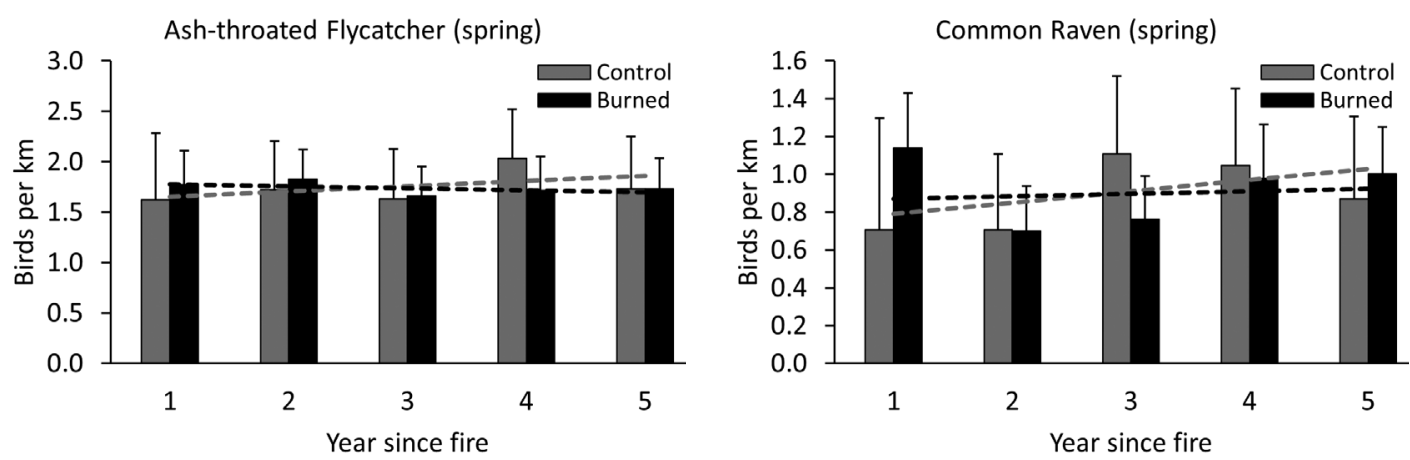

FIGURE 4. Examples of neutral responses to fire (spring/summer): Ash-throated Flycatcher with one of the most even patterns (left), and Common Raven with more variability (right). Both the bars and dashed lines represent responses modeled by mixed-effects regression, the bars with the year as a categorical variable, the lines with the year as a linear variable. Error bars represent $95 \%$ confidence intervals.

\section{PRE-FIRE VS. POST-FIRE}

A total of 129 species qualified (in spring/summer and/or winter) for consideration of changes in the number of atlas squares in which the species was recorded during the 5 years before versus the 5 years after the fires (Table S1). Of the 129, 37 proportionally changed by $>30 \%$ in the number of burned squares in which they were recorded in spring/summer and/or winter (Table S1). Of the 37, 19 increased (15 in spring/summer, 4 in winter) and 18 decreased (3 in spring/summer, 7 in winter, and 8 in both seasons). The strongest positive changes post-fire, according to this metric, tended to be in spring/summer, with the highest number of squares gained by the Rock Wren, followed by the Northern Mockingbird, Black-throated Sparrow, Loggerhead Shrike, and Brewer's Sparrow, while the House and Rock wrens gained the greatest number of squares in winter. They included such species of open habitat as the White-tailed Kite (spring/summer),
Northern Harrier (spring/summer), Say's Phoebe (spring/summer), and Horned Lark (winter). They included two rare riparian species, the Bell's Vireo and Summer Tanager. Two rare visitors, the Common Ground-Dove and Indigo Bunting, appeared in 3 and 6 squares, respectively (representing 5 and 10 records), where they had been unrecorded in the 5 years pre-fire. The species with the highest number of squares lost were the Hutton's Vireo, Brewer's Blackbird, American Robin, and Purple Finch in winter, and Hutton's Vireo, American Robin, and Mountain Chickadee in spring/summer.

In a few cases the comparison with atlas data suggested a response counter to the results of the models. These cases represent complex situations of responses varying by habitat. For example, in its pre-fire habitat of desert-edge scrub, the Blackthroated Sparrow responded negatively and did not recover during the 5 years post-fire (Table 3 ). Yet it also spread in small numbers into some
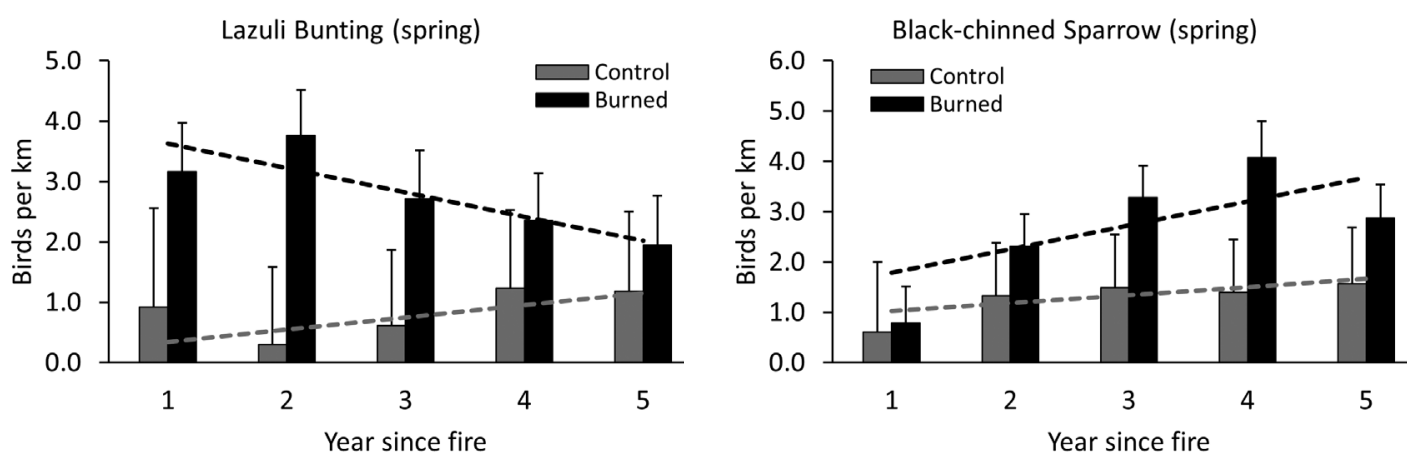

FIGURE 5. Examples of positive responses to fire (spring/summer): Lazuli Bunting, the strongest in second year after fire (left), and Black-chinned Sparrow, strongest in fourth year after fire (right). Both the bars and dashed lines represent responses modeled by mixed-effects regression, the bars with the year as a categorical variable, the lines with the year as a linear variable. Error bars represent $95 \%$ confidence intervals. 

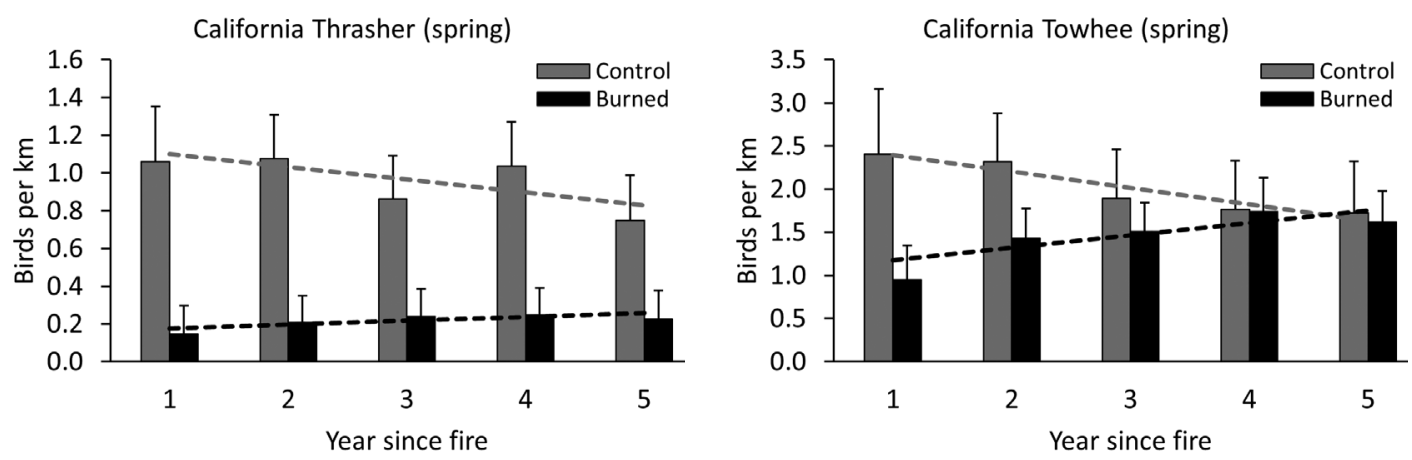

FIGURE 6. Examples of negative responses to fire (spring/summer): California Thrasher, with little recovery (left), and California Towhee, initially negative becoming neutral (right). Both the bars and dashed lines represent responses modeled by mixed-effects regression, the bars with the year as a categorical variable, the lines with the year as a linear variable. Error bars represent $95 \%$ confidence intervals.

areas of burned chaparral where it had formerly been absent, accounting for a notable increase in the number of atlas squares in which it occurred (Table S1).

Isolated small populations of five species that had been confirmed breeding in the Cuyamaca Mountains during field work for the atlas were essentially extirpated after the Cedar Fire, at least temporarily: the Red-breasted Sapsucker, Red-breasted Nuthatch, Hermit Thrush, Goldencrowned Kinglet, and Fox Sparrow. A few summering sapsuckers have been reported from the Cuyamaca Mountains via www.ebird.org since 2010, and the Fox Sparrow apparently recolonized in 2011, being reported subsequently in small numbers annually (from 2004 to 2008, after the departure of migrants, we recorded only one in 2004 and one in 2008). There was a report of a single Hermit Thrush in 2006 and of a single Red-breasted Nuthatch in 2014, but there have been no records of the Golden-crowned Kinglet in the Cuyamaca Mountains during the breeding season since 2003 .

\section{DISCUSSION}

\section{DIVERSITY OF FIRE RESPONSES AND POST-FIRE RECOVERY}

Large-scale fires cause profound changes in the avifauna over short time scales, and in the context of climate warming and drying, these changes may translate into longer-term or permanent shifts in bird distribution and abundance. We categorized the 5-year responses of 92 species to large-scale fires in southern California, providing the first robust quantification for many chaparral species and winter visitors, and identifying the species most at risk from increasing fire.

Responses and trends were more diverse and complex than can be represented by a single perspective. Post-fire models highlighted differences in abundance and trends between burned
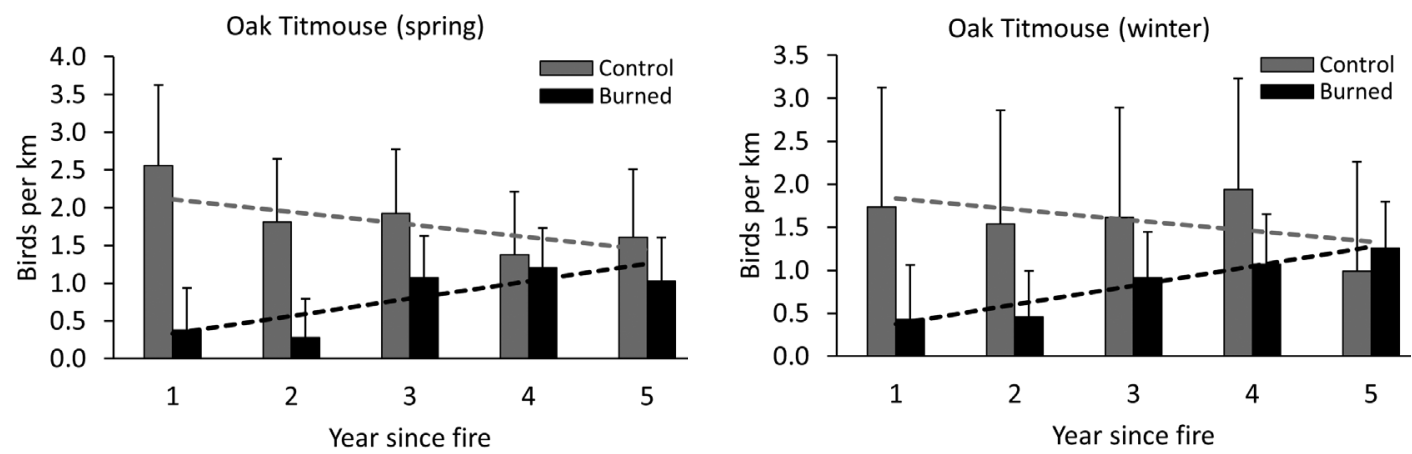

FIGURE 7. Pattern of post-fire change in the Oak Titmouse, in which the trajectory of recovery in spring-summer (left) vs. winter (right) were similar. Both the bars and dashed lines represent responses modeled by mixed-effects regression, the bars with the year as a categorical variable, the lines with the year as a linear variable. Error bars represent $95 \%$ confidence intervals. 

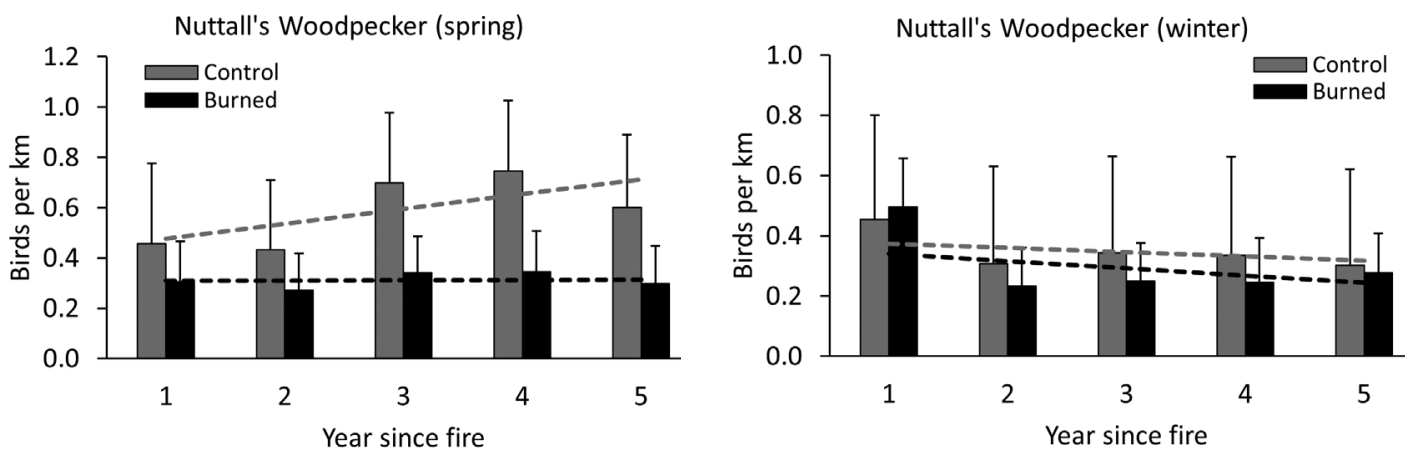

FIGURE 8. Pattern of post-fire change in the Nuttall's Woodpecker, showing a negative response and an increase in control areas relative to burned areas over time in spring/summer (left), contrasted with a more neutral pattern in winter (right). Both the bars and dashed lines represent responses modeled by mixed-effects regression, the bars with the year as a categorical variable, the lines with the year as a linear variable. Error bars represent $95 \%$ confidence intervals.

and unburned routes, while the comparison with pre-fire atlas data highlighted extirpations and colonizations. For example, the models confirm a strongly negative response of the Blue-gray Gnatcatcher even though the number of atlas squares in which it was recorded increased slightly. By contrast, the responses of species too rare for valid modeling are at least suggested by the comparison with pre-fire atlas data. The effects of fire, especially large-scale fire, are difficult to study within the framework of a controlled experimental design. And as Smucker et al. (2005) concluded in Montana, bird species' responses to fire are highly individualistic, depending in part on the fire's severity, time since fire, and, as we identified in this study, elevation and precipitation. Nevertheless, given the magnitude of the biological effects and management implications, it is valuable to identify general response patterns across species and fires.

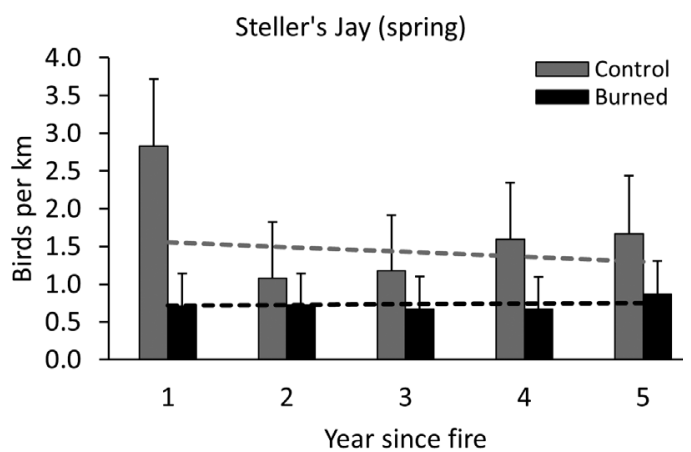

\section{FIRE FOLLOWERS}

Many species benefit from fire, even severe largescale fires. Other studies (e.g., Swanson et al. 2011) have identified benefits to certain species and increases in diversity post-fire. Our set of fire followers, or species responding positively to fire, is large, although less than the set of firesensitive species. Several species (Lazuli Bunting, Costa's Hummingbird, Rock Wren, Lawrence's Goldfinch, and, in winter, Mountain Bluebird) are clearly adapted to exploit recently burned areas, increasing or colonizing in large numbers in the first year or two after the fire (e.g., a count of 215 Lazuli Buntings along just two Cuyamaca survey routes on 28 June 2005).

The biological bases of these positive responses in chaparral need further study, especially for the Lazuli Bunting, which combines nomadism and opportunism with a strictly calendar-driven migration schedule (Unitt 2004). Clarifying these

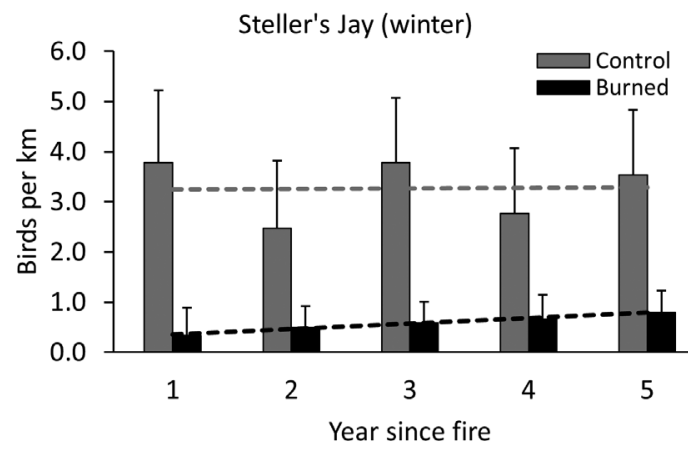

FIGURE 9. Pattern of post-fire change in the Steller's Jay, showing a negative response in spring/summer (left), and an even stronger negative response in winter (right). Both the bars and dashed lines represent responses modeled by mixed-effects regression, the bars with the year as a categorical variable, the lines with the year as a linear variable. Error bars represent $95 \%$ confidence intervals. 

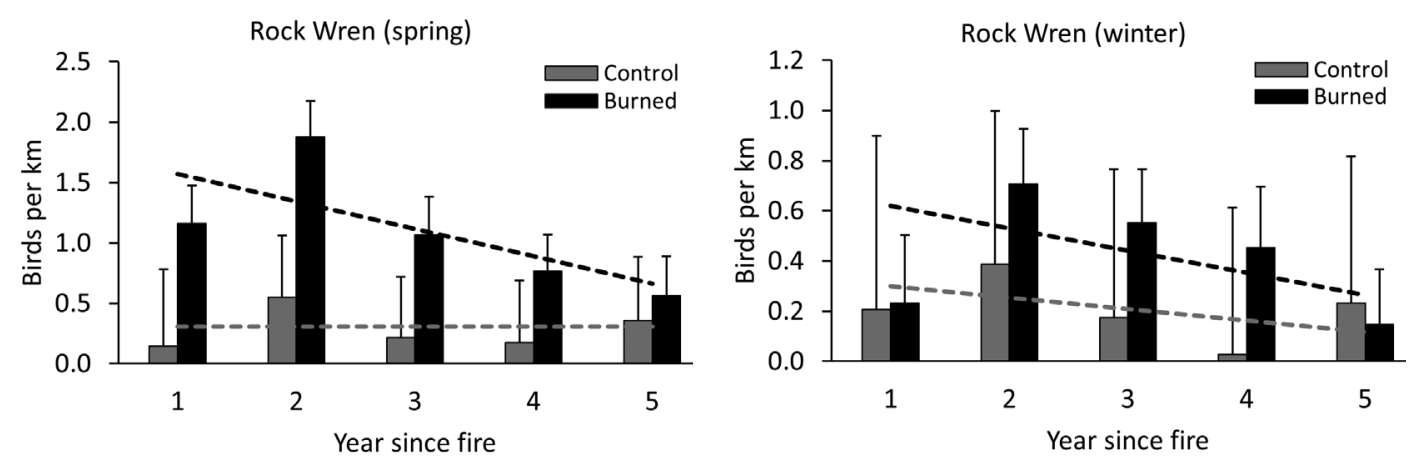

FIGURE 10. Pattern of post-fire change in the Rock Wren, with a strongly positive response and trend of decrease stronger in spring/summer (left), weaker in winter (right). Both the bars and dashed lines represent responses modeled by mixed-effects regression, the bars with the year as a categorical variable, the lines with the year as a linear variable. Error bars represent $95 \%$ confidence intervals.

population dynamics will need research on the reproductive success of birds in burned areas. Nevertheless, we noted the colonization of the Rock Wren of rock outcrops formerly concealed by chaparral or forest, the rapid increase of the Blackchinned Sparrow in the stands of Ceanothus palmeri that replaced the forest in the Cuyamaca Mountains, the feeding of Lawrence's Goldfinch on fire-following wildflowers of the family Boraginaceae, and Costa's Hummingbird feeding on the flowers of fire-following plants such as Penstemon spectabilis and Eriodictyon (Turricula) parryi.

Post-fire avifaunal changes were more pronounced at higher elevations, where the recovery of chaparral was faster, and forest was converted to chaparral. The tendency of fire followers to respond more positively to spikes in precipitation than did fire-sensitive species suggests that opportunism in exploiting burned habitat is correlated with opportunism in exploiting the flush of ephemeral resources during years of ample rain. Numbers of many winter visitors, especially granivores, varied more strongly with the previous winter's precipitation than they did with time since fire. The spike in the number of Lincoln's Sparrows in burned forest in the Cuyamaca Mountains (24 to 39 individuals counted per monthly survey, December 2005-February 2006) was unprecedented for southern California. The species had previously been considered rare as a winter visitor at this elevation (Unitt 2004), and it had not been previously recognized as irruptive or fire-following (Ammon 1995). Similarly, the abundance and spread of the House Wren in winter in burned forest of the Cuyamaca Mountains were beyond historical precedent (38 to 47 individuals counted per monthly survey, December 2005-February 2006, cf. Unitt 2004).
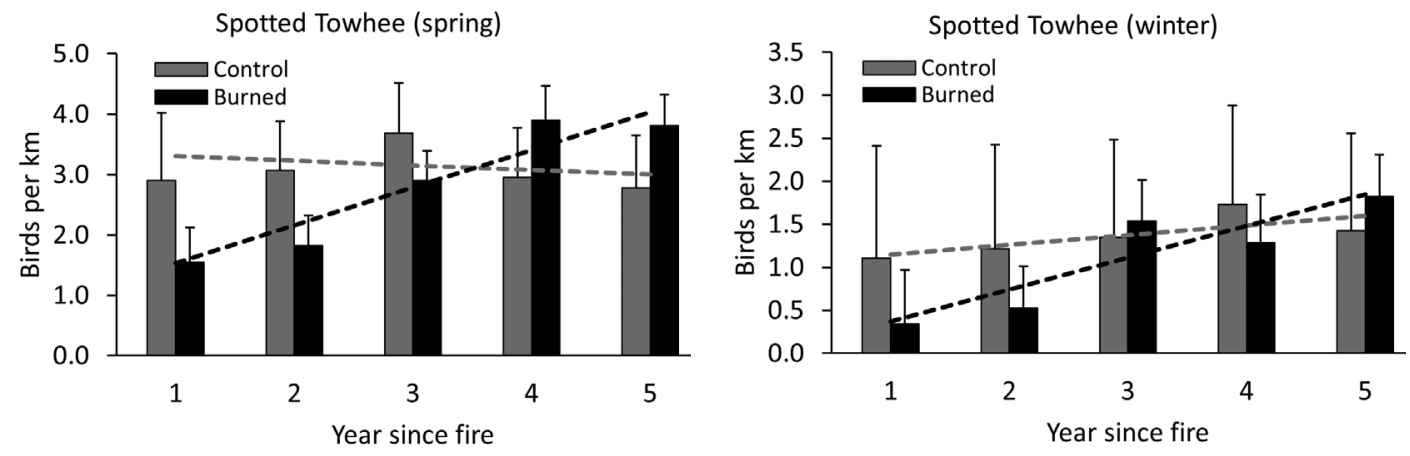

FIGURE 11. Pattern of post-fire change in the Spotted Towhee: a relatively neutral response on the basis of all years averaged, but over 5 years the trend switched from negative to increasingly positive in both spring/summer (left) and winter (right). 

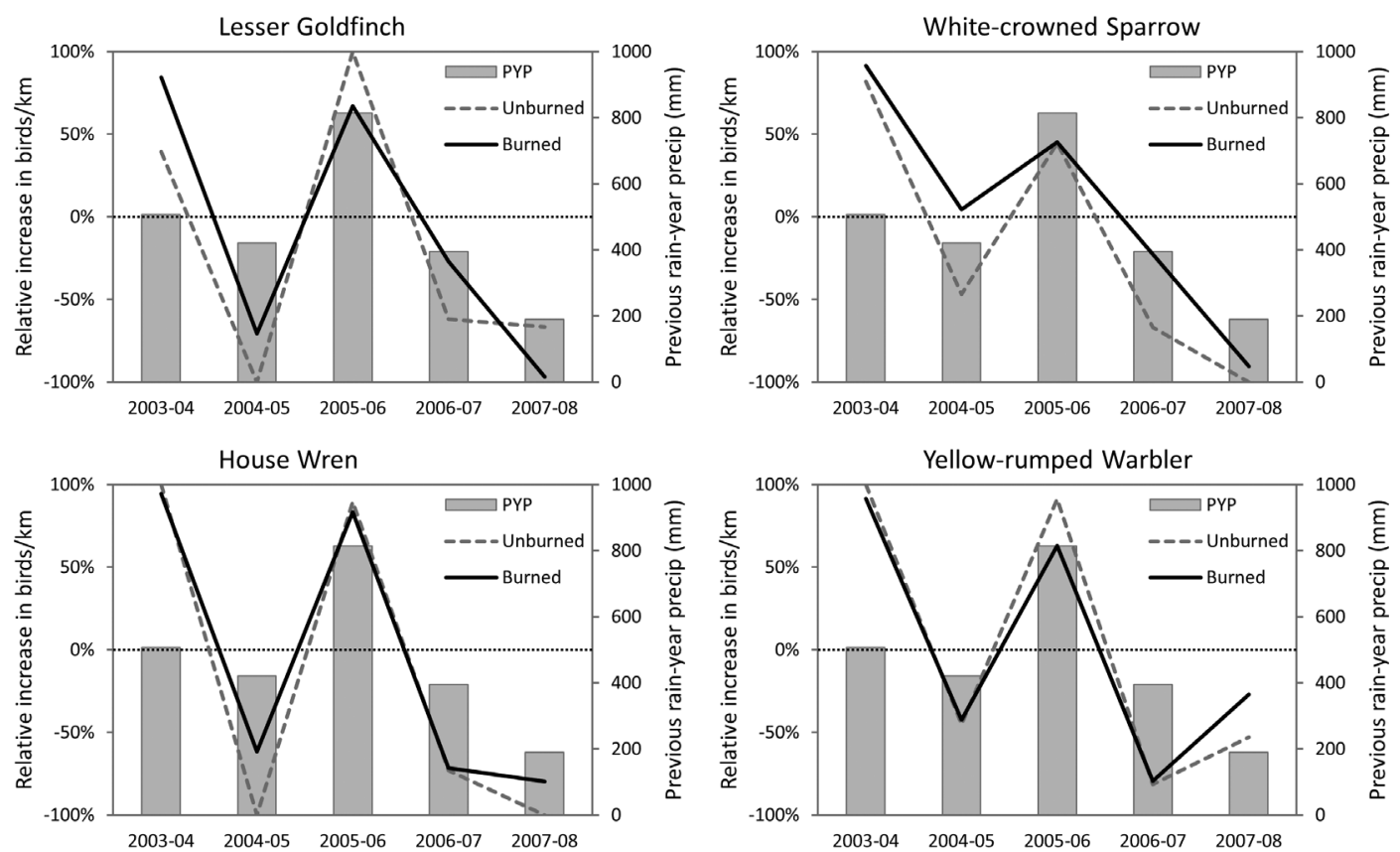

FIGURE 12. Examples of species with winter recovery patterns correlated with precipitation from July to June of the previous year. In 2005-06, the previous year's precipitation (PYP) spiked at $814 \mathrm{~mm}$, correlating with abundance during winter 2005-06, which had increased over that in 2004-05. These four species also showed strong decreases in abundance during the following winter that coincided with a decrease in PYP, and the patterns were similar along both burned and unburned routes.

\section{FIRE NEUTRAL OR RESILIENT}

Species that were categorized as neutral were either indifferent to the fires in that they escaped or avoided the fires, and/or recolonized quickly, or were opportunistic fire followers attracted by the burned habitat, but not strongly enough to be categorized as positive by our analysis. Some species with an initially negative but later positive response appear neutral on the basis of their response over the first 5 years, although study over a longer term could reveal them peaking at an intermediate seral stage. Species with a strong trend of increase post-fire in burned habitat could be considered at least resilient, as could species such as the Rufous-crowned Sparrow, Bell's Sparrow, and Spotted Towhee, which were initially negative but increased in burned areas over prefire abundance, ultimately benefiting from fire.

Neutral responses could be due to more complex underlying patterns, or burned areas meeting some of a species' requirements but not others. For example, the Phainopepla lost a food source in the form of mistletoe, but gained another in the form of elderberry, which increased and grew rapidly in the burned forest on Cuyamaca (pers. obs.). But the increase of elderberry was apparently insufficient to counter the loss of acorns for the Band-tailed Pigeon. How the complexity of ecological relationships relates to the complexity of responses to fire needs much more study.

\section{FIRE SENSITIVE}

Few of the fire-sensitive species had fully recovered in the first 5 years after the fire. The later drier years of our study reversed the recovery of many species. Extended and severe drought, which in southern California can arrest bird reproduction almost totally (Bolger et al. 2005, Langin et al. 2009, Hargrove and Rotenberry 2011a), may cap post-fire recovery.

The species responding most negatively to the fires included many resident species dependent on some degree of shrub or tree cover, such as the Greater Roadrunner, Hutton's Vireo, Steller's Jay, California Thrasher, Pygmy Nuthatch, Cactus Wren, Bewick's Wren, Mountain Chickadee, Oak Titmouse, Bushtit, Wrentit, Purple Finch, and, in winter, Golden-crowned Sparrow and Rubycrowned Kinglet. The strong negative responses of many chaparral and woodland species were not surprising, given their habitat requirements for cover, foraging, and nest substrate that were largely eliminated by the fires.

Many individuals of these species likely also 

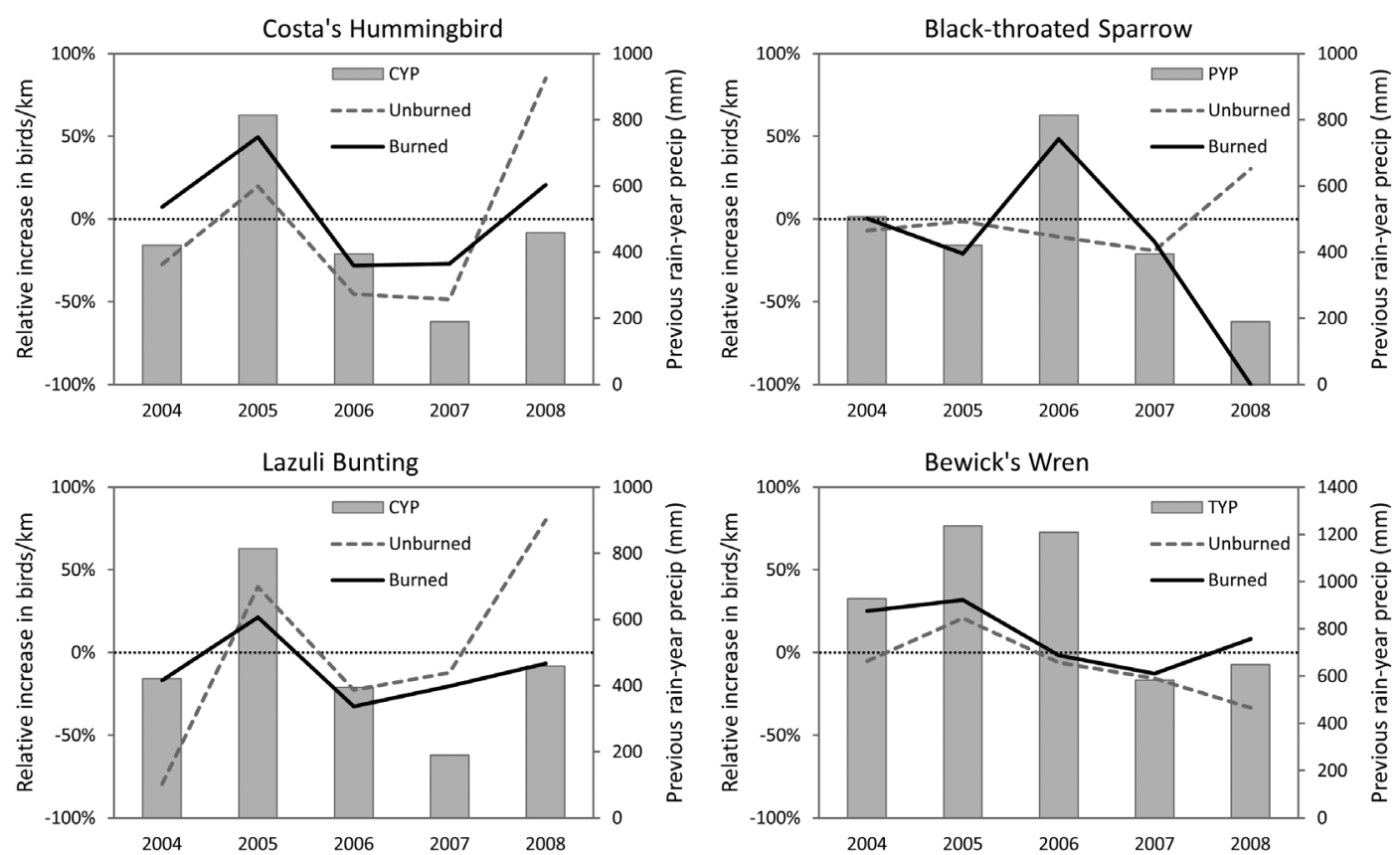

FIGURE 13. Examples of species with spring/summer recovery patterns correlated with precipitation from July to June of the current year (left, Costa's Hummingbird and Lazuli Bunting), with precipitation from July to June of the previous year (upper right, Black-throated Sparrow), and with precipitation during the two years combined (lower right, Bewick's Wren).

perished in the large fires, as has been directly observed in other smaller fires (McClure 1981), and less dispersive species should be slower to recolonize. The common assumption that birds largely escape from fire and then recolonize (e.g., Duncan 1968, Saab and Powell 2005, van Mantgem et al. 2015) is likely true for smallerscale fires and more highly dispersive species, and obviously for migrants not even present during fall fires, such as the Black-chinned Sparrow. But in large-scale, fast-moving fires such as the Cedar Fire, it is not true for weak-flying sedentary species such as the Hutton's Vireo, Bushtit, Wrentit, and California Thrasher. We saw little to no increase over pre-fire numbers of such species on unburned control routes adjacent to the fires, and K. Preston (pers. comm.) confirmed elimination of a marked population of the Wrentit in the Paradise Fire of 2003.

Birds that build open-cup nests in the canopy (e.g., Band-tailed Pigeon, vireos, Western Tanager) recovered poorly in comparison to those that nest in low shrubs or on the ground (e.g., Black-chinned Sparrow, Lark Sparrow, Mountain Quail, California Quail). A leafless canopy offers nests in trees little concealment. Among the most severely affected species are those associated with mixed coniferous forest such as the Mountain Chickadee, Pygmy Nuthatch, Steller's Jay, and Western Tanager. These species were formerly among the most common - and had been since at least 1872, as described by James G. Cooper (1874), the first naturalist to visit the Cuyamaca Mountains. Their rarity after the fire dramatizes the capacity of a large-scale canopy-consuming fire to drive avifaunal change. The negligible germination of conifers on Cuyamaca (Franklin et al. 2006) bodes ill for these bird populations, whose recovery over 5 years was modest to none.

The comparison based on number of atlas squares in which a species was recorded pre- and post-fire illustrates changes in distribution at the rather coarse scale of squares of $-23 \mathrm{~km}^{2}$, but it also highlights changes in localized and rare species. The extirpations from the Cuyamaca Mountains of the small isolated nesting populations of the Red-breasted Sapsucker, Red-breasted Nuthatch, Hermit Thrush, Fox Sparrow, and Golden-crowned Kinglet represented range retractions of those species. The reductions of the White-headed Woodpecker and Brown Creeper came close to truncating the southern margins of the ranges of two more species.

In San Diego, Brewer's Sparrow was rare in 


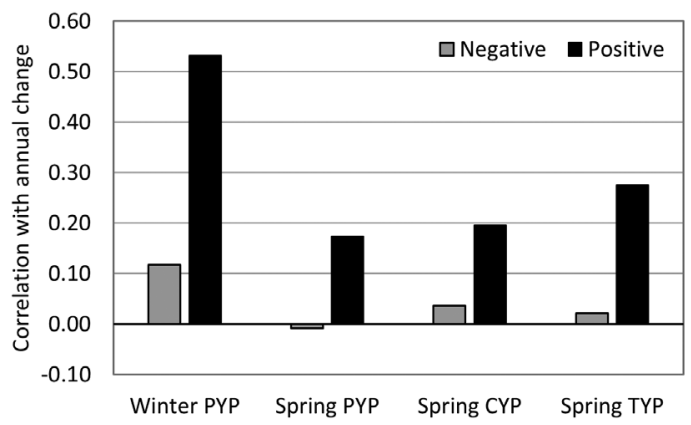

FIGURE 14. Contrast between species responding to fire negatively (gray bars, 35 in winter, 43 in spring) and positively (black bars, 9 in winter, 24 in spring) in average correlation (Pearson product-moment correlation coefficient, $r$ ) between precipitation and annual change in abundance on burned routes. The four combinations of correlations shown are winter species with previous year's precipitation (PYP), spring/summer species with PYP, spring/summer species with current year's precipitation (CYP), and spring/summer species with the two previous years' precipitation combined (TYP).

spring before the fires, and responded negatively, as expected on the basis of studies in its core breeding range (Rotenberry et al. 1999, Miller et al. 2017). The stand of Artemisia tridentata at Ranchita in which Brewer's Sparrow had nested in our study area in 2001 (Unitt 2004), was obliterated the next year in the Pines Fire, with no subsequent recovery. But after the fires, Brewer's Sparrows appeared sparsely in areas of burned chaparral, and even burned forest, leading to an instance of apparent hybridization with the Blackchinned Sparrow (Unitt 2007).

The susceptibility of the Cactus Wren to fire in southern California was anticipated by Rea and Weaver (1990) with respect to the coastal subspecies sandiegensis and demonstrated by its collapse after the Laguna Fire of 1993 and the Witch Creek Fire of 2007 (Hamilton et al. 2011); we saw that vulnerability reflected in the inland subspecies anthonyi. Even though desert-edge species use semi-open habitat, many of their responses were negative (Greater Roadrunner, Ladder-backed Woodpecker, Cactus Wren, Blackthroated Sparrow, Scott's Oriole). Thus the role of fire in eliminating key habitat components such as mature cactus or yucca highlights the greater importance of floristic variables in limiting desertedge species (Hargrove and Rotenberry 2011b).

\section{COMPARISONS TO OTHER STUDIES}

Our findings bolstered the profile of many species already described as positively affected by fire, such as the Lazuli Bunting (Leidolf et al. 2007, Greene et al. 2014), Rock Wren (Saab and Powell 2005), Mountain Bluebird (Saab and Powell 2005), Black-chinned Sparrow (Grinnell and Miller 1944, Burridge 1995), and Chipping Sparrow (Middleton 1998, Saab and Powell 2005), as well as some negatively affected, such as the Pygmy Nuthatch (Brawn and Balda 1988), Steller's Jay (Saab and Powell 2005), and California Thrasher (Cody 2012). The extirpation of the Red-breasted Nuthatch from Cuyamaca adds to its reputation for negative responses to fire (Kirkpatrick et al. 2006).

The importance of fire in the biology of many of the fire followers we identified, however, has been generally underappreciated, especially in the case of the Lawrence's and Lesser goldfinches and Costa's Hummingbird. Though the opportunism of the Lazuli Bunting (Greene et al. 2014) and irruptiveness of the Black-chinned Sparrow (Tenney 1997) were known to some extent, the numbers occupying burned habitat in San Diego County were so large as to imply shifting of the population on a large scale. Also in San Diego County, Mendelsohn et al. (2008) found the Lazuli Bunting to be the most positively affected by fire at their study site, burned in the Otay Fire of 2003. Though Leidolf et al. (2007) wrote that the fire-following they studied in the Lazuli Bunting was "local in nature and cannot be readily extrapolated," we found that it can. Those authors suggested avoidance of cowbird parasitism as a driver of the Lazuli Bunting's preference for burned habitat, although we suspect it is the flush of suitable insect prey feeding on the new growth of plants in the spring following the fire, paralleling the attraction of deer to feed on newly sprouting growth (Botta 2017).

We also found congruence with general patterns reported in other studies of coniferous/ woodland habitat, such as in the Sierra Nevada, where Raphael et al. (1987) found increases in ground- and brush-foraging birds and decreases in arboreal birds, but see Raphael et al. (2018) for longer-term responses. In the Sierra Nevada, Bagne and Purcell (2011) also found negative responses by the Hutton's and Warbling vireos. The Dusky Flycatcher, thought to benefit from reduced over-story elsewhere (Kroll and Haufler 2007), was our strongest positive responder proportionally, though in low numbers (reaching 5 to 9 per survey cycle in the last year of study on Cuyamaca).

However, many other fires whose effects have been studied in both woodland and chaparral have 
tended to be controlled, smaller, and/or of lower intensity. Thus such studies have tended to find weaker changes in species composition and more rapid recovery than we observed (e.g., England 1995). The much greater size and severity of the San Diego County fires may also explain why some prescribed fires in the Sierra Nevada have found responses contrary to our results, such as post-fire increases over 6 years in the Western Flycatcher, Brown Creeper, and American Robin (Bagne and Purcell 2011). Within 2 years after severe stand-replacing fires in coniferous forest in Montana and Wyoming, Hutto (1995) identified the Hairy Woodpecker, Olive-sided Flycatcher, American Robin, and Dark-eyed Junco as fire followers, whereas in the Cuyamaca Mountains we found these species responding negatively or neutrally. The difference may be due to the fires Hutto studied being embedded in a large region of similar forest, whereas the relative isolation of the Cuyamaca Mountains inhibits dispersal of coniferous forest species, and the limited extent of this habitat in southern California implies that source populations are lower.

We found a negative response for the Western Bluebird, which has shown positive responses elsewhere (Saab and Powell 2005). The bluebird's response is likely dependent on the severity of the fire and its effect on the number of snags and live trees remaining (Guinan et al. 2008). We suspect similar factors contributed to other negative responses that contrasted with previous studies, especially in the cases of the Olive-sided Flycatcher (Altman and Sallabanks 2012), Brown Creeper (Poulin et al. 2013), and Hermit Thrush (Dellinger et al. 2012).

A lag longer than the 5 years of our study may be needed for other species to respond positively, as seen in the Fox Sparrow in the Sierra Nevada (Bock and Lynch 1970), in the Western WoodPewee in southeastern Arizona (Kirkpatrick et al. 2006), and in other species shown to be fire followers in the Rocky Mountains in somewhat older burned forests, such as the Northern Flicker and Steller's Jay (Hutto 1995). Nevertheless, we found that the initially negative response by the Spotted Towhee reversed to positive in 4 years, supporting the positive association with severe fire reported by Kirkpatrick et al. (2006).

A notable exception to our finding of a more negative response to fire than reported generally is the Ash-throated Flycatcher, which Saab and Powell (2005) noted as negative over multiple studies in pinyon/juniper woodland but we found to be neutral with respect to fire.
Though our study was inadequate for thorough analysis of grassland species, we noted the Horned Lark colonizing burned areas formerly covered with dense chaparral, a positive response paralleling that reported for this species by Mendelsohn et al. (2008) and Earnst et al. (2009).

Analyzing the influx of Lazuli Buntings into 800 ha of burned oak woodland in Utah, Leidolf et al. (2007) quantified a spillover of the influx into unburned habitat within $1 \mathrm{~km}$ of the burned patch. A similar spillover evidently affected some of our unburned routes, at least in the case of strong fire followers such as the Lazuli Bunting and Black-chinned Sparrow. This lack of independence of the burned and unburned routes likely depressed our measures of response to the fire, both positive and negative. Thus the effects of the fire may be stronger than our analysis implies.

\section{SPECIES AT RISK FROM INCREASING FIRES}

Species most at risk from increasing fires are those with a strong negative response combined with slow recovery. A shift to a drier climate and more frequent fires will clearly reduce the numbers and ranges of many species, even those relatively common, such as the Pygmy Nuthatch, Mountain Chickadee, and California Thrasher. However, many species that appear resilient to fire in the short term could also be at risk through reduced survival and reproduction post-fire. Robinson et al. (2013) pointed out the importance of refuges or unburned patches, which could assist recovery if the patches are large enough. These might also be enhanced by fire-management strategies. Yet the diversity of fire responses across species does not necessarily imply that creating a landscape with a heterogeneous fire history is the best management strategy (Taylor et al 2012). Any species dependent on extensive mature chaparral may be threatened by fires increasing beyond the frequency of the 3-5 years many species of chaparral shrubs take to reach reproductive maturity (Barro and Conard 1991).

The most immediate risk is to species of birds requiring later successional stages but whose population is already small or whose distribution is limited. Birds requiring later successional stages may be expected to fare poorly where post-fire succession is impaired by invasive species, habitat fragmentation, and climate change. The Gray Vireo, a rare species with a patchy distribution, already faces multiple stressors that may lower its resiliency to fire (Hargrove and Unitt 2014, 2017), reducing its ability to recolonize. In southern California, numerous coniferous woodland 
species have a "sky island" distribution, putting them at risk of range retraction from increasing fires and drought. This risk is particularly high for species like the White-headed Woodpecker and Brown Creeper, which reach the southern limit of their range among small sky islands (e.g., Kratter 1992) that are more likely to burn completely. Extirpations of local populations not only erode a species' range, they reduce its genetic diversity, long-term viability, and evolutionary potential (Hampe and Petit 2005). The extirpations of the five species from the Cuyamaca Mountains represented northward retractions of their breeding ranges. All five were recent colonists, possibly in response to the increasing density of the forest before the fire (Unitt and Hargrove 2018). From 1932 to the time of the Cedar Fire, the density of conifers in the Cuyamaca Mountains had increased by a factor of 2.6 (Goforth and Minnich 2008).

\section{Fire ANd AVIfaunal Change}

In the case of fire-sensitive species with discontinuous distributions, fires as massive as the ones we studied, followed by habitat conversion, could result in retraction of ranges by large discrete steps. By the end of the century, the total area of California burned annually is expected to increase by $9-15 \%$ above the historical norm (Lenihan et al. 2008). Thus we may expect biogeographic patterns to be increasingly altered by fire, but with complex interactions. Furthermore, post-fire recovery depends not only on the frequency and intensity of fires; it can also be strongly affected by the surrounding landscape. As urbanization dominates ever more of the southern California landscape, there is less opportunity for recolonization by non-urban species after large-scale fires and increasing opportunity for colonization by invasive species (Syphard et al. 2006). Since 2003, large-scale wildfires have continued to burn southern California nearly annually, in some cases burning hundreds of square kilometers burned only a few years previously (http://www.fire. ca.gov/fire_protection/fire_protection_fire_info_ redbooks_2015). Where chaparral has replaced forest, some formerly abundant species such as the Mountain Chickadee and Pygmy Nuthatch are now rare. Cumulatively, and within the context of climate change and urbanization, such fires have the capability to drive not only short-term variations but also longer-term and permanent range shifts and avifaunal change.

\section{ACKNOWLEDGMENTS}

We thank our supporters who have made this research possible: California State Parks, Joint Fire Science Program, U.S. Forest Service, Cleveland National Forest, and Blasker Environment Grant Program of the San Diego Foundation, and especially Paul D. Jorgensen, Thomas A. Oberbauer, and Kirsten Winter. Thanks to the volunteers who dedicated over 8000 hours of field time: Barbara Anderson, Maryanne Bache, Joe Barth, Thomas A. Blackman, Rich and Susan Breisch, Sean Buchanan, Stephen D. Cameron, Kevin B. Clark, Andrea Neal Compton, Jim Determan, Kathy Estey, Gary Grantham, Edward C. Hall, Hank Ingersoll, Paul D. Jorgensen, Ann and Tom Keenan, Kerry Kenwood, Carol McKie Manning, Gillian Moreland, Philip K. Nelson, Barbara Orr, Nuri B. Pierce, Wayne Pray, Geoffrey L. Rogers, Jay Rourke, Robert Sanger, Kathie Satterfield, Herb Stone, Mary Beth Stowe, Lee E. Taylor, Robert Thériault, Frank L. Unmack, Kenneth L. Weaver, James K. Wilson, Kirsten Winter, Anne Wright, and James O. Zimmer. Thank you to Marcia Narog of the Forest Fire Laboratory, Pacific Southwest Research Station, U.S. Forest Service, for her contribution to our ecological and botanical perspective. We thank Colleen Handel, Kris Preston, W. David Shuford, Robert E. Gill Jr., and anonymous referees for their incisive reviews.

It is our pleasure and honor to dedicate this paper to the memory of Paul D. Jorgsensen (1946-2012), former ecologist for California State Parks' Colorado Desert District. Paul's efforts, both in the field and as an administrator, were key to the completion of the San Diego County Bird Atlas, to our coverage of the area burned in the Pines Fire, both within and without Anza-Borrego Desert State Park, and for the extension of our study to include Cuyamaca Rancho and Palomar Mountain state parks. We miss him dearly.

\section{Literature Cited}

Altman, B., and Sallabanks, R. 2012. Olive-sided Flycatcher (Contopus cooperi), in The Birds of North America Online (A. Poole, ed.), no. 502. Cornell Lab. Ornithol., Ithaca, NY; doi 10.2173/bna.502.

Ammon, E. M. 1995. Lincoln's Sparrow (Melospiza lincolnii), in The Birds of North America Online (A. Poole and F. Gill, eds.), no. 191. Cornell Lab. Ornithol., Ithaca, NY; doi 10.2173/bna.191.

Bagne, K. E., and Purcell, K. L. 2011. Short-term responses of birds to prescribed fire in fire-suppressed forests of California. J. Wildl. Mgmt. 75:10511060; doi 10.1002/jwmg.128.

Barro, S. C., and Conard, S. G. 1991. Fire effects on Cal- 
ifornia chaparral systems: An overview. Environ. Int. 17:135-149; doi 10.1016/0160-4120(91)90096-9.

Bates, D., Maechler, M., Bolker, B., and Walker, S. 2014. lme4: Linear mixed-effects models using Eigen and S4. R package version 1.1-7; CRAN.Rproject.org/package $=1$ me4.

Bock, C. E., and Lynch, J. F. 1970. Breeding bird populations of burned and unburned conifer forest in the Sierra Nevada. Condor 72:182-189.

Bolger, D. T., Patten, M. A., and Bostock, D. C. 2005. Avian reproductive failure in response to an extreme climatic event. Oecologia 142:398-406; doi 10.1007/s00442-004-1734-9.

Botta, R. 2017. Mule deer (Odocoileus hemionus), in San Diego County mammal atlas (S. Tremor, D. Stokes, W. Spencer, J. Diffendorfer, H. Thomas, S. Chivers, and P. Unitt, eds.), pp. 338-342. Proc. San Diego Soc. Nat. Hist. 46.

Brawn, J. D., and Balda, R. P. 1988. The influence of silvicultural activity on ponderosa pine forest bird communities in the southwestern United States. Bird Conserv. 3:3-21.

Burnham, K. P., and Anderson, D. R. 2002. Model Selection and Multimodel Inference. Springer, New York.

Burridge. B. 1995. Sonoma County Breeding Bird Atlas. Madrone Audubon Soc., Santa Rosa, CA.

Cody, M. L. 2012. California Thrasher (Toxostoma redivivum), in The Birds of North America Online (A. Poole, ed.), no. 323. Cornell Lab. Ornithol., Ithaca, NY; doi 10.2173/bna.323.

Cooper, J. G. 1874. Animal life of the Cuyamaca Mountains. Am. Nat. 8:14-18; doi 10.1086/271250.

Dellinger, R., Wood, P. B., Jones, P. W., and Donovan, T. M. 2012. Hermit Thrush (Catharus guttatus), in The Birds of North America Online (A. Poole, ed.), no. 261. Cornell Lab Ornithol., Ithaca, NY; doi 10.2173/bna.261.

Duncan, D. A. 1968. Food of California Quail on burned and unburned central California foothill rangeland. Calif. Fish and Game 54:127-127.

Earnst, S. L., Newsome, H. L., LaFramboise, W. L., and LaFramboise, N. 2009. Avian response to wildfire in interior Columbia basin shrubsteppe. Condor 111:370-376; doi 10.1525/cond.2009.080109.

England, A. S. 1995. Avian community organization along a post-fire age gradient in California chaparral. Ph.D. dissertation, Univ. of Calif., Davis.

Fiske, I., and Chandler, R. 2011. Unmarked: An R package for fitting hierarchical models of wildlife occurrence and abundance. J. Stat. Software 43(10): 1-23; doi 10.18637/jss.v043.i10.

Franklin, J., Spears-Lebrun, L. A., Deutschman, D. H., and Marsden, K. 2006. Impact of a high-intensity fire on mixed evergreen and mixed conifer forests in the Peninsular Ranges of southern California, USA. For. Ecol. Mgmt. 235:18-29; doi 10.1016/j. foreco.2006.07.023.

Goforth, B. R., and Minnich, R. A. 2008. Densification, stand-replacement wildfire, and extirpation of mixed conifer forest in Cuyamaca Rancho State Park, southern California. For. Ecol. Mgmt. 256:36-45; doi 10.1016/j.foreco.2008.03.032.

Greene, E., Muehter, V. R., and Davison, W. 2014. Lazuli Bunting (Passerina amoena), in The Birds of North America Online (A. Poole, ed.), no. 232. Cornell Lab. Ornithol., Ithaca, NY; doi 10.2173/bna.232.

Grinnell, J., and Miller, A. H. 1944. The distribution of the birds of California. Pac. Coast Avifauna 27.

Guinan, J. A., Gowaty, P. A., and Eltzroth, E. K. 2008. Western Bluebird (Sialia mexicana), in The Birds of North America Online (A. Poole, ed.), no. 510. Cornell Lab. Ornithol., Ithaca, NY; doi 10.2173/ bna. 510 .

Hamilton, R. A., Proudfoot, G. A., Sherry, D. A., and Johnson, S. 2011. Cactus Wren (Campylorhynchus brunneicapillus), in The Birds of North America Online (A. Poole, ed.), no. 558. Cornell Lab. Ornithol., Ithaca, NY; doi 10.2173/bna.558.

Hampe, A., and Petit, R. J. 2005. Conserving biodiversity under climate change: The rear edge matters. Ecol. Lett. 8:461-467; doi 10.1111/j.14610248.2005.00739.x.

Hargrove, L., and Rotenberry, J. T. 2011a. Breeding success at the range margin of a desert species: Implications for a climate-induced elevational shift. Oikos 120:1568-1576; doi 10.1111/j.16000706.2011.19284.x.

Hargrove, L., and Rotenberry, J. T. 2011b. Spatial structure and dynamics of breeding bird populations at a distribution margin, southern California. J. Biogeogr. 38:1708-1716; doi 10.1111/j.13652699.2011.02525.x.

Hargrove, L., and Unitt, P. 2014. Gray Vireo (Vireo vicinior) status assessment and nest monitoring to investigate causes of decline in California. Nongame Wildlife Program Rep. 2014-01. Calif. Dept. of Fish and Wildlife, Sacramento; www.dfg.ca.gov/wildlife/ nongame/publications/.

Hargrove, L., and Unitt, P. 2017. Poor reproductive success of Gray Vireos in a declining California population. J. Field Ornithol. 88:16-29; doi 10.1111/ jofo. 12189.

Hutto, R. L. 1995. Composition of bird communities following stand-replacement fires in northern Rocky Mountain (U.S.A.) conifer forests. Conserv. Biol. 9:1041-1058; doi 10.1046/j.15231739.1995.9051033.x-i1.

Keeley, J. E., and Zedler, P. H. 2009. Large, high-intensity fire events in southern California shrublands: Debunking the fine-grain age patch model. Ecol. Appl. 19:69-94; doi 10.1890/08-0281.1.

Keeley, J. E., Fotheringham, C. J., and Morais, M. 1999. Reexamination of fire suppression impacts on brushland fire regimes. Science 284:1829-1832; doi 10.1126/science.284.5421.1829.

Keeley, J. E., Brennan, T., and Pfaff, A. H. 2008. Fire severity and ecosystem responses following crown fires in California shrublands. Ecol. Appl. 18:15301546; doi 10.1890/07-0836.1. 
Kirkpatrick, C., Conway, C. J., and Jones, P. B. 2006. Distribution and relative abundance of forest birds in relation to burn severity in southeastern Arizona. J. Wildl. Mgmt. 70:1005-1012; doi 10.2193/0022-541X(200 6)70[1005:DARAOF $] 2.0 . \mathrm{CO} ; 2$.

Kratter, A. W. 1992. Montane avian biogeography in southern California and Baja California. J. Biogeogr. 19:269-283; doi 10.2307/2845451.

Kroll, A. J., and Haufler, J. B. 2007. Evaluating habitat quality for the Dusky Flycatcher. J. Wildl. Mgmt. 71:14-22; doi 10.2193/2005-452.

Langin, K. M., Sillett, T. S., Yoon, J., Sofaer, H. R., Morrison, S. A., and Ghalambor, C. K. 2009. Reproductive consequences of an extreme drought for Orange-crowned Warblers on Santa Catalina and Santa Cruz islands, in Proceedings of the 7th California Islands Symposium (C. C. Damiani and D. K. Garcelon, eds.), pp. 293-300. Inst. Wildlife Studies, Arcata, CA.

Lawrence, G. E. 1966. Ecology of vertebrate animals in relation to chaparral fire in Sierra Nevada foothills. Ecology 47:278-291; doi 10.2307/1933775.

Leidolf, A., and Bissonette, J. A. 2009. The effects of fire on avian communities: Spatio-temporal attributes of the literature 1912-2003. Int. J. Wildland Fire 18:609-622; doi 10.1071/WF08019.

Leidolf, A., Nuttle, T., and Wolfe, M. L. 2007. Spatially scaled response of a Lazuli Bunting population to fire. W. N. Am. Nat. 67:1-7; doi 10.3398/1527-0904(2007)67[1:SSROAL]2.0.CO;2.

Lenihan, J. M., Bachelet, D., Neilson, R. P., and Drapek, R. 2008. Response of vegetation distribution, ecosystem productivity, and fire to climate change scenarios for California. Clim. Change 87:S215S230; doi 10.1007/s10584-007-9362-0.

McClure, H. E. 1981. Some responses of resident animals to the effects of fire in a coastal chaparral environment in southern California. Trans. W. Sect. Wildl. Soc. 17:86-99.

McKenzie, D., Gedalof, Z., Peterson, D. L., and Mote, P. 2004. Climatic change, wildfire, and conservation. Conserv. Biol. 18:890-902; doi 10.1111/j.15231739.2004.00492.x.

Mendelsohn, M. B., Brehme, C. S., Rochester, C. J., Stokes, D. C., Hathaway, S. A., and Fisher, R. N. 2008. Responses in bird communities to wildland fires in southern California. Fire Ecol. 4:63-82; doi 10.4996/fireecology.0402063.

Middleton, A. L. 1998. Chipping Sparrow (Spizella passerina), in The Birds of North America Online (A. Poole and F. Gill, eds.), no. 334. Cornell Lab. Ornithol., Ithaca, NY; doi 10.2173/bna.334.

Miller, R. A., Bond, L., Migas, P. N., Carlisle, J. D., and Kaltenecker, G. S. 2017. Contrasting habitat associations of sagebrush-steppe songbirds in the intermountain West. W. Birds 48:35-55; doi 10.21199/ WB481.3.

Moriarty, D. J. 2013. A twenty-year investigation of the effects of fire on a coastal sage scrub bird community. W. Birds 44:2-16.
Moriarty, D. J., Farris, R. E., Noda, D. K., and Stanton, P. A. 1985. Effects of fire on a coastal sage scrub bird community. Southwest. Nat. 30:452-453; doi $10.2307 / 3671284$.

Poulin, J.-F., D’Astous, E., Villard, M.-A., Hejl, S. J., Newlon, K. R., McFadzen, M. E., Young, J. S., and Ghalambor, C. K. 2013. Brown Creeper (Certhia americana), in The Birds of North America Online (A. Poole, ed.), no. 669. Cornell Lab. Ornithol., Ithaca, NY; doi 10.2173/bna.669.

Raphael, M. G., Morrison, M. L., and Yoder-Williams, M. P. 1987. Breeding bird populations during 25 years of postfire succession in the Sierra Nevada. Condor 89:614-626.

Raphael, M. G., Airola, D. A., Falxa, G. A., Harris, R. D., and Stine, P. A. 2018. Breeding bird changes during 50 years of post-fire succession in the Sierra Nevada, in Trends and Traditions: Avifaunal change in western North America (W. D. Shuford, R. E. Gill Jr., and C. M. Handel, eds.), pp. 400-418. Studies of Western Birds 3. Western Field Ornithologists, Camarillo, CA; doi 10.21199/SWB3.21.

Rea, A. M., and Weaver, K. L. 1990. The taxonomy, distribution, and status of coastal California Cactus Wrens. W. Birds 21:81-126.

Regan, H.M., Hierl, L.A., Franklin, J., and Deutschman, D. H. 2006. San Diego Multiple Species Conservation Program covered species prioritization. Report to Calif. Dept. of Fish and Game, Sacramento; nrm.dfg.ca.gov/FileHandler.ashx?DocumentID=15634.

Robinson, N. M., Leonard, S. W. J., Ritchie, E. G., Bassett, M., Chia, E. K., Buckingham, S., Gibb, H., Bennett, A. F., and Clarke, M. F. 2013. Refuges for fauna in fire-prone landscapes: Their ecological function and importance. J. Appl. Ecol. 50:1321-1329; doi 10.1111/1365-2664.12153.

Rotenberry, J. T., Patten, M. A., and Preston, K. L. 1999. Brewer's Sparrow, in the Birds of North America Online (A. Poole and F. Gill, eds.), no. 390. Cornell Lab. Ornithol., Ithaca, NY; doi 10.2173/ bna.390.

Saab, V. A., and Powell, H. D. W. 2005. Fire and avian ecology in North America: Process influencing pattern. Studies Avian Biol. 30:1-13.

Smucker, K. M., Hutto, R. L., and Steele, B. M. 2005. Changes in bird abundance after wildfire: Importance of fire severity and time since fire. Ecol. Appl. 15:1535-1549; doi 10.1890/04-1353.

Stanton, P. A. 1986. Comparison of avian community dynamics of burned and unburned coastal sage scrub. Condor 88:285-289.

Swanson, M. E., Franklin, J. F., Beschta, R. L., Crisafulli, C. M., Dellasalla, D. A., Hutto, R. L., Lindenmayer, D. B., and Swanson, F. J. 2011. The forgotten stage of forest succession: Early-successional ecosystems on forest sites. Front. Ecol. Environ. 9:117-125; doi 10.1890/090157.

Syphard, A. D., Franklin, J., and Keeley, J. E. 2006. Simulating the effects of frequent fire on southern California coastal shrublands. Ecol. Appl. 16:1744- 
1756; doi 10.1890/1051-0761(2006)016[1744:ST EOFF]2.0.CO;2.

Taylor, R. S., Watson, S. J., Nimmo, D. G., Kelly, L. T., Bennett, A. F., and Clarke, M. F. 2012. Landscapescale effects of fire on bird assemblages: Does pyrodiversity beget biodiversity? Diversity Distrib. 18:519529; doi 10.1111/j.1472-4642.2011.00842.x.

Tenney, C. R. 1997. Black-chinned Sparrow (Spizella atrogularis), in The Birds of North America Online (A. Poole and F. Gill, eds.), no. 270. Cornell Lab. Ornithol., Ithaca, NY; doi 10.2173/bna.270.

Unitt, P. 2004. San Diego County bird atlas. Proc. San Diego Soc. Nat. Hist. 39.

Unitt, P. 2007. An apparent hybrid Brewer's × Blackchinned Sparrow. W. Birds 38:280-288.

Unitt, P., and Hargrove, L. 2018. Southward and downslope extensions of breeding ranges of birds in southern California, in Trends and traditions: Avifaunal change in western North America (W. D. Shuford,
R. E. Gill Jr., and C. M. Handel, eds.), pp. 85-115. Studies of Western Birds 3. Western Field Ornithologists, Camarillo, CA; doi 10.21199/SWB3.4.

Van Mantgem, E. F., Keeley, J. E., and Witter, M. 2015. Faunal responses to fire in chaparral and sage scrub in California, USA. Fire Ecol. 11:128-148; doi 10.4996/fireecology. 1103128 .

Wirtz, W. O. 1979. Effects of fire on birds in chaparral. Cal-Neva Wildlife Trans., pp. 114-124.

Wirtz, W. O. 1982. Postfire community structure of birds and rodents in southern California chaparral, in Proceedings of the symposium on dynamics and management of Mediterranean-type ecosystems (C. E. Conrad and W. C. Oechel, eds.), pp. 241-246. Gen. Tech. Rep. PSW-GTR-58, USDA For. Serv., Pacific Southwest Forest and Range Exp. Stn., Berkeley, CA; www.fs.fed.us/psw/publications/documents/ psw_gtr058/psw_gtr058.pdf.

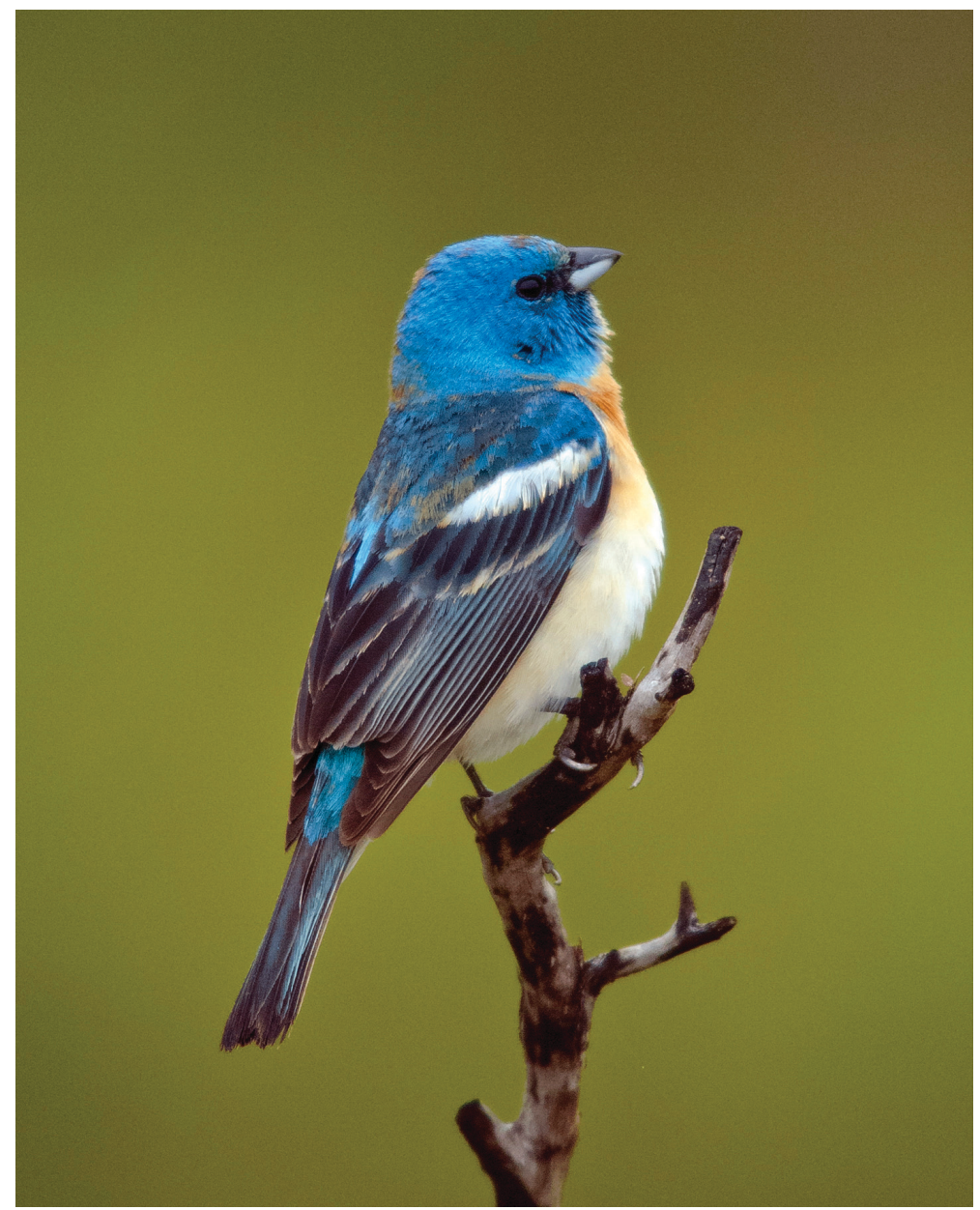

Lazuli Bunting on snag of chaparral burned in the Cedar Fire, central San Diego County, California.

Photo by Jack C. Daynes. 